\begin{tabular}{|l|l|}
\hline 2. To: (Receiving Organization) & $\begin{array}{l}\text { 3. From: Coriginating Organization) } \\
\text { Nuclear Physics and Shielding }\end{array}$ \\
\hline 5. Proj./Prog./Dept./Div.: & 6. Cog. Engr.: \\
West Valley & V. E. Roetman \\
\hline
\end{tabular}

8. Originator Remarks:

Documentation of Shielding Calculations for West Valley Vitrification Cell

4. Related EDT No.:

7. Purchase Order No.:

9. Equip./Component No.:

10. System/Bldg./Facility:

11. Receiver Remarks:

12. Major Assm. Dwg. No.:

13. Permit/Permit Application No.:

14. Required Response Date:

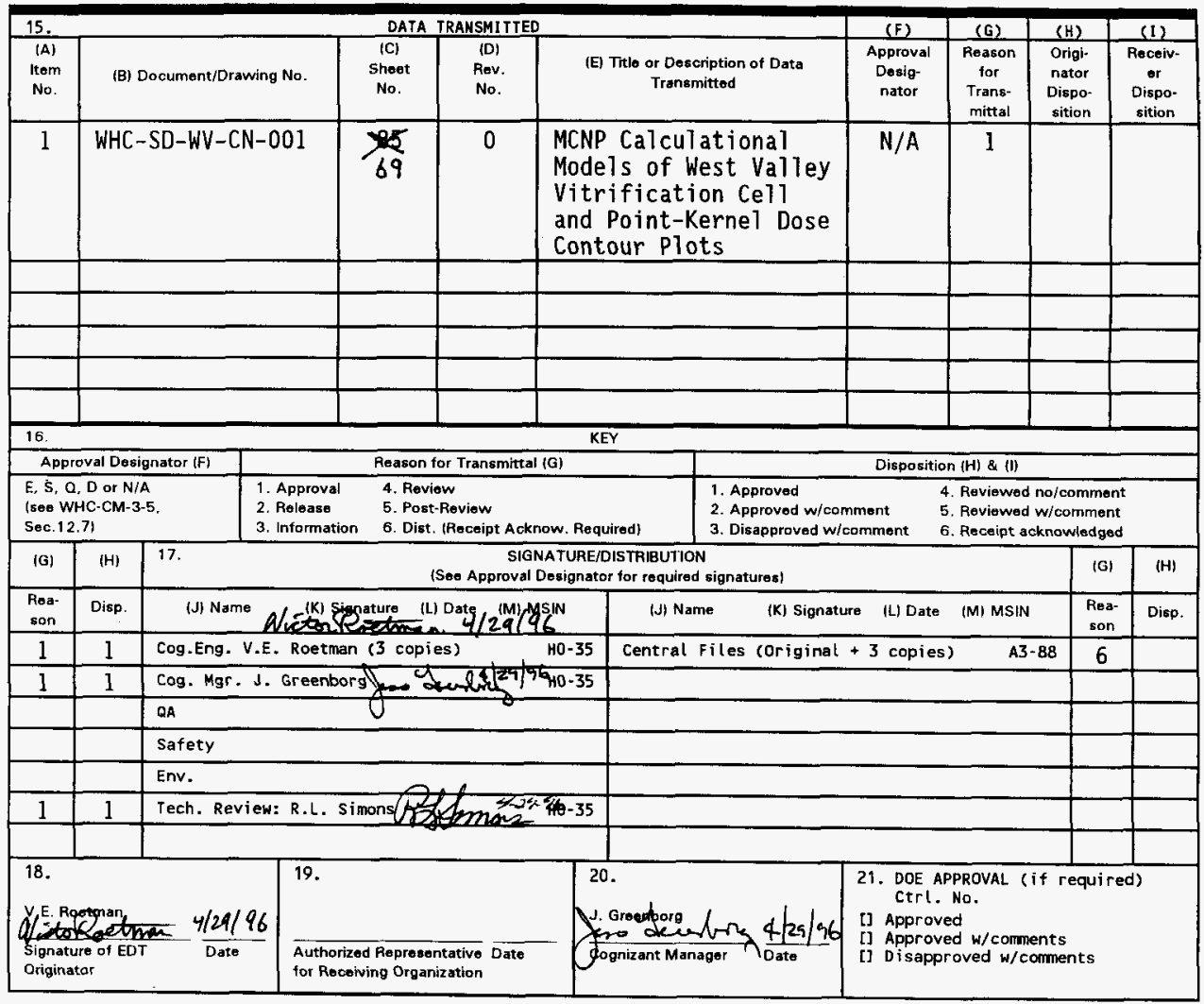

BD-7400-172-2 (04/94) GEF097 


\section{MCNP Calculational Models of West Valley Vitrification Cell and Point-Kernel Dose Contour Plots}

\section{E. Roetman}

Westinghouse Hanford Company, Richland, WA 99352

U.S. Department of Energy Contract DE-AC06-87RL10930
EDT/ECN: 603240
Org Code: $8 M 730$
UC: 507
B\&R Code: EX3133000
Charge Code: YX332
Total Pages: 69

Key Words: West Valley, Vitrification, Dose Rates, Shielding

Abstract: This documents the radiation physics model and dose rate calculations for the West Valley vitrificaton cell.

TRADEMARK DISCLAIMER. Reference herein to any specific comercial product, process, or service by trade name, trademark, manufacturer, or otherwise, does not necessarily constitute or imply its endorsement, recommendation, or favoring by the United States Government or any agency thereof or its contractors or subcontractors.

Printed in the United States of America. To obtain copies of this document, contact: WHC/BCS Document Control Services, P.O. Box 1970, Mailstop H6-08, Richland WA 99352, Phone (509) 372-2420; Fax (509) 376-4989.
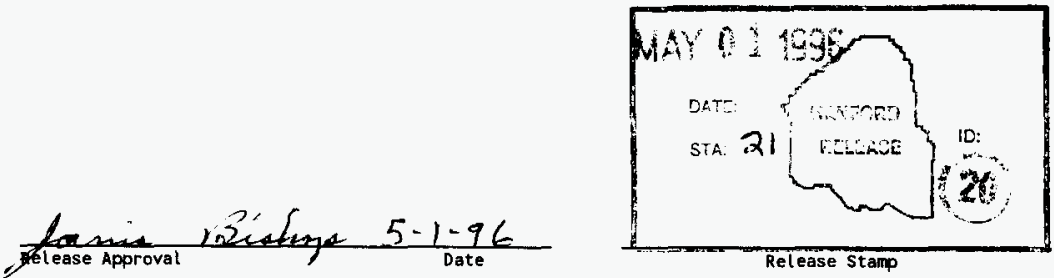

\section{Approved for Public Release}


WHC-SD-WV-CN-001, Rev.0

Page 2 of 69

\section{MCNP CALCULATIONAL MODELS OF WEST VALLEY VITRIFICATION CELL AND POINT-KERNEL DOSE CONTOUR PLOTS}

\subsection{INTRODUCTION}

The West Valley Vitrification Facility cell contains the concentrator tank, the feed tank, the melter, and up to twenty glass filled canisters. It is shielded with 48 inch concrete walls at the lower elevations, and by 34 and 26 inch walls at the upper elevations, as well as two steel doors. MCNP (see References 1 and 2) physics calculational models were constructed that included these sources and the shields. The MCNP models used for streaming calculations (e.g. the crane maintenance room, HEPA filter room) were developed from these models.

The dose rates inside and outside the cell were computed using the pointkernel option of MCNP, resulting in dose contour plots at four elevations for two source configurations (fifteen canisters, and twenty canisters).

\subsection{METHODOLOGY}

Figures 1 through 7 show three dimensional Sabrina (See Reference 3) plots of the MCNP calcuiational model.

The cell was modeled using the construction drawings of the facility (see Reference 4). The MCNP origin $(0,0,0)$ for all physical dimensions was located at the north-west corner of the inside of the cell at EL 100'. The modeled cell internal width at elevation $91.44 \mathrm{~cm}$ (EL 103' or 3 feet above ground level) is $1036 \mathrm{~cm}$, and the internal length is $1920 \mathrm{~cm}$. At MCNP elevation $1051.56 \mathrm{~cm}$ (drawing EL 134.5') the cell model is $1158.24 \mathrm{~cm}$ wide, and $1975.8 \mathrm{~cm}$ long (internal). The model places the crane maintenance room (CMR) door at $2083.04 \mathrm{~cm}$ from the north cell wall (at EL 134.5'), and the iron door is $22.86 \mathrm{~cm}$ thick. The concrete walls at the lower elevation are modeled as $121.92 \mathrm{~cm}$ thick, and the walls at the upper elevation are $66.04 \mathrm{~cm}$ thick. Not included in the calculational model was the equipment on the west half of the cell. This is conservative because this equipment would provide some additional shielding. 
WHC-SD-WV-CN-001, Rev. 0

Page 3 of 69

Some other conservative assumptions made in this calculation are:

The canisters are $100 \%$ filled instead of $85 \%$.

The source has an extra $15 \%$ curie concentration due to process uncertainties.

The concrete density is assumed to be at $1421 \mathrm{~b} / \mathrm{ft}^{3}$.

No reinforcing bar and very little structural steel was modeled in the walls.

A11 the vessels and containers were assumed full.

The maximum height of a canister on the crane is EL 130' 8.125".

A full MCNP photon source description includes the concentrator tank, the feed tank, the melter, and twenty glass filled canisters. Four canisters are located in the turntable underneath the melter, eight in the pit storage rack, two in the welder station, one in the decontamination station, four on the transfer cart, and one on the crane against the east wall with the canister top at the maximum elevation of $935.04 \mathrm{~cm}$. The other source configuration is the same except it excludes the canister on the crane and the four canisters on the cart, making the total number of canisters fifteen. The source strength and spectrum are specified in the MCNP input files (see the listing of the input files in Attachment A).

The photon source was obtained using the information provided by West Valley in Tables 1 and 2, where the melter feed source is described in curies $/ \mathrm{cm}^{3}$ for each nuclide in Table 1 , and the glass source is described in curies/g for each nuclide in Table 2. The volume and densities of each source type is as follows:

$\begin{array}{lll}\text { Feed Tank and Concentrator: } & \begin{array}{l}\text { Material Density: } \\ \text { Volume: }\end{array} & 1.3 \mathrm{~g} / \mathrm{cm}^{3} \\ & & 1.897 \times 10^{7} \mathrm{~cm}^{3} \\ \text { Melter Glass: } & \begin{array}{l}\text { Material Density: } \\ \text { Volume: }\end{array} & 2.7 \mathrm{~g} / \mathrm{cm}^{3} \\ & & 8.587 \times 10^{5} \mathrm{~cm}^{3} \\ \text { Canister Glass: } & \text { Material Density: } & 2.7 \mathrm{~g} / \mathrm{cm}^{3} \\ & \text { Volume: } & 2.298 \times 10^{5} \mathrm{~cm}^{3}\end{array}$

The photon energy spectrum and the total photons per second per unit volume (for feed) or unit mass (for glass) of material was generated with ISOSHLD (see Reference 5 and Attachment B). The total photons $/ g$ of the $g l$ ass and total photons $/ \mathrm{cm}^{3}$ of the feed were multiplied by the total mass of the glass and the total volume of the feed to get the total source strength in photons $/ \mathrm{sec}$, which was $3.4 \times 10^{16}$ photons $/ \mathrm{sec}$. 
Table 1. Radionuclide concentration in curies for $1 \mathrm{~cm}^{3}$ of waste feed.

\begin{tabular}{|c|c|c|}
\hline $\mathrm{Fe}-55 \quad 1.20-7$ & Eu-154 $4.96-5$ & Th-232 $1.38-9$ \\
\hline Co-60 2.95-7 & Eu-155 8.68-6 & Th-234 $6.72-10$ \\
\hline $\mathrm{Ni}-59 \quad 8.92-8$ & T1-207 7.92-9 & $\mathrm{Pa}-231 \quad 1.28-8$ \\
\hline $\mathrm{Ni}-63 \quad 6.18-6$ & Tl-208 2.52-9 & $\mathrm{Pa}-233 \quad 1.98-8$ \\
\hline Se-79 $3.11-9$ & Pb-209 $1.81-10$ & $\mathrm{~Pa}-234 * 6.72-10$ \\
\hline$S r-90 \quad 4.89-3$ & Pb-211 7.95-9 & U-232 5.54-9 \\
\hline$\gamma-90 \quad 4.89-3$ & $\mathrm{~Pb}-2127.02-9$ & U-233 $7.61-9$ \\
\hline$Z r-93 \quad 2.29-7$ & $\mathrm{Bi}-2117.95-9$ & U-234 3.64-9 \\
\hline $\mathrm{Nb}-93^{*} 1.71-7$ & $\mathrm{Bi}-212 \quad 7.02-9$ & U-235 7.97-11 \\
\hline TC-99 $1.02-7$ & $B i-213 \quad 1.81-10$ & $U-236 \quad 2.34-10$ \\
\hline Ru-106 $2.14-10$ & Po-212 4.50-9 & $U-238 \quad 6.72-10$ \\
\hline$R h-106 \quad 1.94-10$ & Po-213 $1.77-10$ & $N p-2367.98-9$ \\
\hline Pd-107 9.28-9 & Po-215 7.95-9 & $\mathrm{Np}-237 \quad 1.98-8$ \\
\hline $\mathrm{Cd}-113^{*} 1.49-6$ & Po-216 7.02-9 & Np-239 $2.92-7$ \\
\hline$S n-121^{*} 1.35-8$ & At-217 $1.81-10$ & Pu-236 $7.10-10$ \\
\hline$S n-126 \quad 8.77-8$ & $R n-2197.95-9$ & Pu-238 6.67-6 \\
\hline Sb-125 $1.36-6$ & $R n-220 \quad 7.02-9$ & $P u-239 \quad 1.37-6$ \\
\hline Sb-126 $1.23-8$ & $\mathrm{Fr}-221 \quad 1.81-10$ & Pu-240 $1.01-6$ \\
\hline$S b-126^{\star} 8.77-8$ & $\mathrm{Fr}-223 \quad 1.10-10$ & $P u-241 \quad 5.09-5$ \\
\hline Te-125* $3.51-7$ & Ra-223 7.95-9 & $\mathrm{Pu}-242 \quad 1.37-9$ \\
\hline$I-129 \quad 1.52-10$ & Ra-224 7.02-9 & Am-241 $4.51-5$ \\
\hline Cs-134 5.89-7 & $\mathrm{Ra}-225 \quad 1.81-10$ & Am-242 $2.42-7$ \\
\hline Cs-135 $1.38-7$ & Ra-228 $1.33-9$ & Am-242* 2.43-7 \\
\hline C5-137 5.38-3 & $A C-225 \quad 1.81-10$ & Am-243 2.92-7 \\
\hline $\mathrm{Ba}-137 * 5.09-3$ & AC-227 7.95-9 & $\mathrm{Cm}-242 \quad 2.01-7$ \\
\hline Ce-144 2.62-12 & $A C-228 \quad 1.33-9$ & Cm-243 $9.76-8$ \\
\hline $\operatorname{Pr}-144 \quad 2.63-12$ & Th-227 7.83-9 & $\mathrm{Cm}-244 \quad 5.12-6$ \\
\hline Pm-146 4.28-9 & Th-228 7.02-9 & Cm-245 7.43-10 \\
\hline $\mathrm{Pm}-147 \quad 1.51-5$ & Th-229 $1.81-10$ & $\mathrm{Cm}-246 \quad 8.50-11$ \\
\hline Sm-151 $6.78-5$ & Th-230 4.94-11 & \\
\hline Eu-152 2.26-7 & Th-231 7.97-11 & * isomeric state \\
\hline
\end{tabular}


Table 2. Radionuclide concentration in curies for 1 gram of glass.

\begin{tabular}{|c|c|c|}
\hline $\mathrm{Fe}-55 \quad 2.93-7$ & Eu-155 2.13-5 & Th-234 $1.65-9$ \\
\hline $\mathrm{C} 0-607.25-7$ & Tा-207 1.94-8 & $\mathrm{Pa}-231 \quad 3.14-8$ \\
\hline $\mathrm{Ni}-59 \quad 2.19-7$ & T1-208 6.19-9 & $\mathrm{Pa}-233 \quad 4.85-8$ \\
\hline $\mathrm{Ni}-63 \quad 1.52-5$ & $\mathrm{~Pb}-209$ 4.44-10 & $\mathrm{Pa}-234^{*} 1.65-9$ \\
\hline Se-79 $7.29-9$ & Pb-211 1.95-8 & U-232 $1.36-8$ \\
\hline$S r-90 \quad 1.20-2$ & $\mathrm{~Pb}-212 \quad 1.72-8$ & U-233 $1.87-8$ \\
\hline$Y-90 \quad 1.20-2$ & $\mathrm{Bi}-211 \quad 1.95-8$ & U-234 8.95-9 \\
\hline$Z r-93 \quad 5.63-7$ & Bi-212 $1.72-8$ & $U-235 \quad 1.96-10$ \\
\hline Nb-93* 4.19-7 & $\mathrm{Bi}-213 \quad 4.44-10$ & $U-236 \quad 5.73-10$ \\
\hline Tc-99 2.25-7 & Po-212 $1.11-8$ & $U-238 \quad 1.65-9$ \\
\hline$R u-106 \quad 4.75-10$ & Po-213 4.34-10 & $N p-236 \quad 1.96-8$ \\
\hline $\mathrm{Rh}-106 \quad 4.76-10$ & Po-215 1.95-8 & $\mathrm{Np}-237 \quad 4.85-8$ \\
\hline$P d-107$ 2.28-8 & Po-216 1.72-8 & $N p-2397.17-7$ \\
\hline$C d-113 * 3.30-6$ & At-217 $4.44-10$ & Pu-236 $1.74-9$ \\
\hline $5 n-121 * 3.32-8$ & $R n-219 \quad 1.95-8$ & $\mathrm{Pu}-238 \quad 1.64-5$ \\
\hline$S n-126 \quad 2.15-7$ & $\mathrm{Rn}-220 \quad 1.72-8$ & Pu-239 3.36-6 \\
\hline Sb-125 3.35-6 & $\mathrm{Fr}-221 \quad 4.44-10$ & Pu-240 2.47-6 \\
\hline Sb-126 3.01-8 & $\mathrm{Fr}-223 \quad 2.69-10$ & $P u-241 \quad 1.25-4$ \\
\hline$S b-126^{*} 2.15-7$ & Ra-223 1.95-8 & $\mathrm{Pu}-242 \quad 3.36-9$ \\
\hline Te-125* $8.21-7$ & Ra-224 1.72-8 & Am-241 $1.11-4$ \\
\hline Cs-134 1.42-6 & $R a-225 \quad 4.44-10$ & Am-242 5.94-7 \\
\hline Cs-135 3.34-7 & $\mathrm{Ra}-228 \quad 3.26-9$ & Am-242* $5.97-7$ \\
\hline Cs-137 $1.30-2$ & Ac- -225 4.44-10 & Am-243 7.17-7 \\
\hline $\mathrm{Ba}-137 * 1.23-2$ & $A C-227 \quad 1.95-8$ & $\mathrm{Cm}-242 \quad 4.93-7$ \\
\hline Ce-144 $6.44-12$ & $A c-228 \quad 3.26-9$ & $\mathrm{Cm}-243 \quad 2.40-7$ \\
\hline $\mathrm{Pr}-144 \quad 6.45-12$ & Th-227 $1.92-8$ & $\mathrm{Cm}-244 \quad 1.26-5$ \\
\hline Pm-146 $1.05-8$ & Th-228 $1.72-8$ & Cm-245 $1.82-9$ \\
\hline Pm-147 3.71-5 & Th-229 4.44-10 & Cm-246 2.09-10 \\
\hline$S m-151 \quad 1.67-4$ & Th-230 $1.21-10$ & \\
\hline Eu-152 5.56-7 & Th-231 $1.96-10$ & * isomeric state \\
\hline Eu-154 1.22-4 & Th-232 3.39-9 & \\
\hline
\end{tabular}


The material compositions used in the MCNP input files are shown in Table 3. A11 percentages are mass density unless otherwise specified.

An array of 2000 point detectors was positioned at each of four levels in order to calculate point-kernel dose rate information for obtaining contour plots. The elevations of the detectors are $91.44 \mathrm{~cm}$ (EL 103'), $403.86 \mathrm{~cm}$ (EL 113.25'), $822.96 \mathrm{~cm}$ (EL 127'), and $1051.56 \mathrm{~cm}$ (EL 134.5'). The dose rates were calculated using MCNPH, the Hanford version of MCNP, which includes the ability to tally large arrays of point detectors in point kernel mode. The dose contour plots were generated from the MCNPH output data using the visualization package PV-Wave (see Reference 6).

\subsection{RESULTS}

Figures 8 through 19 show the dose contour plots at each of the four levels with the fuil canister configuration, and at the upper two levels with the canister configuration without the cart or crane sources. There are two plots for each level, one detailing the dose rates inside of the cell, and one detailing the dose rates outside the cell, making a total of twelve plots. The dimension scale is in $\mathrm{cm}$ according to the MCNP coordinates. The view is a plan view of the cell with north being to the left. The dose rate contour lines are labeled as rem or mrem, referring to $\mathrm{rem} / \mathrm{hour}$ and mrem/hour respectively. The plot elevation is labeled in feet where the ground level is at EL $100^{\prime}$.

These dose plots were generated using PV-Wave and were calculated from the dose point arrays generated from MCNPH point-kernel calculations. The contour ine positions were calculated from linear interpolation along an array of the $\log$ of the dose values.

The dose rates at three specific points were also calculated with the pointkerne? method. All three of these points were at the same $X$ value as the center of the canister on the crane. The top of the crane canister is at $935.04 \mathrm{~cm}$.

1) Dose at MCNP location $x, y, z=1318.02,1453.17,1051.56$

(EL 134.5', 114.18" east of the outside upper wall)

$3.00 \mathrm{mrem} / \mathrm{hr}$ 
WHC-SD-WV-CN-001, Rev.0

Page 8 of 69

2) Dose at MCNP location $x, y, z=1318.02,1878.17,1051.56$

(EL 134.5', 281.5" east of the outside upper wa11)

$1.44 \mathrm{mrem} / \mathrm{hr}$

3) Dose at MCNP location $x, y, z=1318.02,1453.17,853.44$

(EL 128', 114.18" east of the outside upper wal1)

$6.50 \mathrm{mrem} / \mathrm{hr}$

The first two points are above the canister by $116.52 \mathrm{~cm}$. The third point is horizontally and vertically in line with the top section of the canister.

There are some anomalies in the dose contours plots. These are caused by:

1) inherent assumptions in the buildup factors used in point-kernel calculations. At large angles through a shield, point-kernel assumes the line-of-sight shield thickness. For some geometric situations, this will cause lower calculated values closer to the shield wall than farther away.

2) Statistical uncertainties due to very low dose rates, such as around corners. At these low values the statistical uncertainties of the Monte Carlo sampling of the source are large, therefore causing some uncertainties in the contour lines.

3) The penetrations in the CMR doorway allow some of the points a view of the sources with very little shielding, thereby reading high dose values. Because this view may be at an angle, but the dose points lie on a horizontal plane, the dose lines may appear to be isolated islands.

4) The PV-Wave package uses a linear interpolation approximation to calculate the position of a dose line between detector positions, which may give results slightly different than what MCNP would caiculate.

The point-kernel method of calculating dose rates is especially well suited to direct shielding, i.e. a source, a shield (such as a wall) and a detector where the line between the source and the detector lies perpendicular to the wail. The approximations tend to give lower values when the line between the source and the detector becomes more parallel to the wall. This is because the point-kernel method assumes a thick shield in these cases, and does not consider the possibility of a particle approaching the wall at a shallow 
angle, and reflecting at a sharp angle, straight through the wall and into a detector.

Figure 12 shows an example of the limitations of point-kernel calculations for complex geometrical situations. According to the figure, the dose rate increases when moving away from the wall at the top of the page (on the east side of the building) eventually exceeding $.25 \mathrm{mrem} / \mathrm{h}$. This exaggerated effect is coming from the source that is on the crane, against the upper wall. Because the upper wall is much thinner than the lower walls, the dose rates due to that source will be higher than the other sources in the cell. Close to the wall, the line between the canister on the crane and the detector goes at a sharp angle through the wall, where point-kernel assumes a thick shield and therefore low dose rates. As the detector moves further out from the wall, the angle grows more shallow, and the apparent shield becomes thinner, and the dose rates increase. At larger distances from the wa11, the pointkernel results will be similar to monte-carlo results, but at distances close to the wall, the dose rates will read lower from point-kernel than they would in a full monte carlo calculation. Although the dose rates probably would increase as one moves farther from the wall, this effect is exaggerated in the point-kernel calculation, and the dose rates closer to the wall are underestimated. The increased dose rates will come from scattered photons from the upper source through the thin wall, which is not taken into consideration in the point-kernel model.

Likewise the higher elevation dose rates would be better estimated using full Monte-Carlo calculations, because the scattering of the lower level source radiation in the thin upper walls is not taken into consideration in the point-kernel buildup factors.

These input files are archived on CFS under the directory / $93586 / w v /$ source/ and are named inp5.CraneOnWall and inp5. NoCart.

\subsection{CONCLUSIONS}

These MCNP calculational models of the vitrification cell were used as a starting point for many of the streaming calculations that were done with MCNP. These models include two input files that differ only from the description of two different source configurations. These calculational models were also utilized for point kernel calculations of dose rates beyond the bulk walls and within the cell. 
WHC-SD-WV-CN-001, Rev.0

Page 10 of 69

\subsection{REFERENCES}

1. Breismeister, J. F., Editor, "MCNP - A General Monte Carlo Code N-Particle Transport Code, Version 4A," LA-12625, Los Alamos National Laboratory, Los Alamos, New Mexico (1993).

2. Carter, L. L., "Certification of MCNP Version 4A for WHC Computer Platforms," ECN 186710, January, 1994.

3. Van Riper, K. A., "SABRINA Users's Guide," LA-UR-93-3696, Los Alamos National Laboratory, Los Alamos, New Mexico (1993).

4. Drawings provided by West Valley Nuclear Services, West Valley, New York:

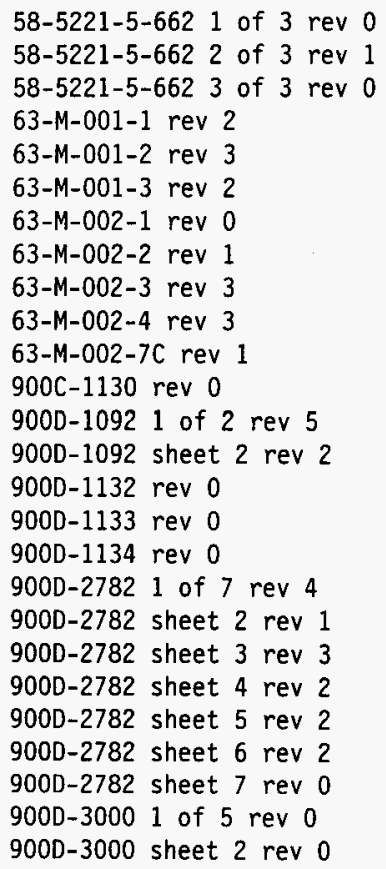




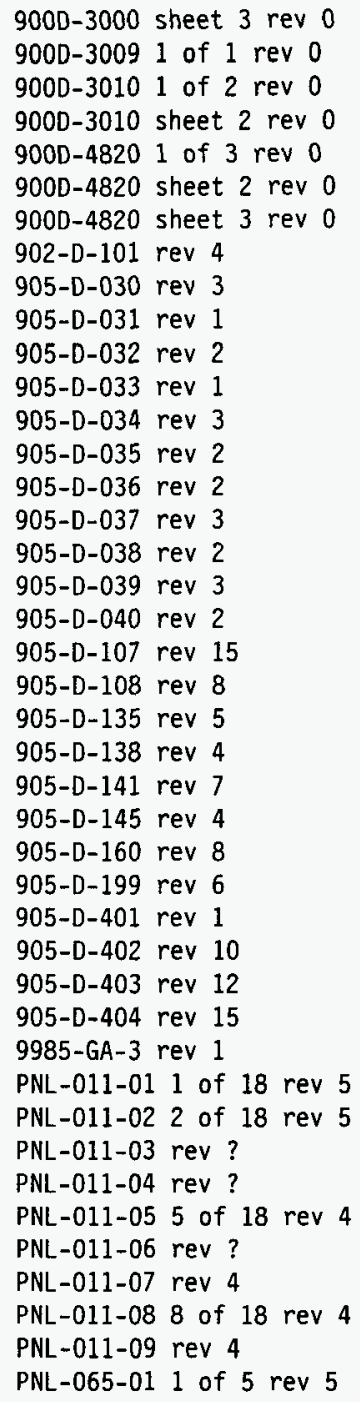


WHC-SD-WV-CN-001, Rev.0

Page 12 of 69

PNL-065-02 2 of 5 rev 1

PNL-065-03 3 of 5 rev 1

PNL-065-04 4 of 5 rev 1

PNL-065-05 5 of 5 rev 3

5. Israel 0. Lourant, "Validation of ISOSHLD-PC on the IBM PC (PS-2/AT/XT), EDT-109878, WHC-SD-SQA-CSWD-303, Rev. 0, February 1990.

6. PV-Wave Version $5.0 \bigcirc 1994$ by Visual Numerics, Inc. 
WHC-SD-WV-CN-001, Rev.0

Page 13 of 69

\subsection{COMPUTATION}

The source strength was calculated as follows:

The input for ISOSHLD was the given source description for one gram of glass and the output was the total photons per second, as well as the photons per second of each energy group. This was also done for the source description of one cubic centimeter of feed waste. The ISOSHLD output files are included in Attachment B.

The output shows one gram of glass to be $5.326 \times 10^{8}$ photons $/ \mathrm{sec}^{\star} \mathrm{g}$. One cubic centimeter of feed is $2.105 \times 10^{8}$ photons $/ \mathrm{sec}^{\star} \mathrm{cm}^{3}$.

The volume of one canister of glass is $8.512 \times 10^{5} \mathrm{~cm}^{3}$, and the density of the glass is $2.7 \mathrm{~g} / \mathrm{cm}^{3}$. This makes the mass of one canister to be $2.298 \times 10^{6} \mathrm{~g}$. The source strength in photons/sec of one canister is $2.298 \times 10^{6} \mathrm{~g} * 5.326 \times 10^{8}$ photons $/ \mathrm{sec}^{*} \mathrm{~g}=1.224 \times 10^{15}$ photons $/ \mathrm{sec}$. The melter glass region has a volume of $8.587 \times 10^{5} \mathrm{~cm}^{3}$ and a mass of $2.319 \times 10^{6} \mathrm{~g}$. This makes a source strength of $1.235 \times 10^{15}$ photons/sec. The feed tank and the concentrator tanks both have a source volume of $1.897 \times 10^{7} \mathrm{~cm}^{3}$, and with a source strength of $2.105 \times 10^{8}$ photons $/ \mathrm{sec}^{\star} \mathrm{cm}^{3}$, the source strength of each tank is $3.993 \times 10^{15}$ photons $/ \mathrm{sec}$.

The total source strength in the cell is the feed/concentrator strength * $2+$ the melter strength +20 * the canister strength; $2 * 3.993 \times 10^{15}+1.235 \times 10^{15}$ $+20 * 1.224 \times 10^{15}=3.370 \times 10^{16}$ photons $/ \mathrm{sec}$. This source strength is increased by $15 \%$ for a conservative calculation, making the total source strength in the cell to be $3.88 \times 10^{16}$ photons $/ \mathrm{sec}$. This matches the source card wgt of $3.91+16$ (the slight difference is from using more significant figures in this calculation than in the previous calculation).

Each individual source is assigned a fraction of the total source weight based on the number of photons/sec from that source, and is assigned a weighted energy distribution which also was generated with ISOSHLD. 
WHC-SD-WV-CN-001, Rev.0

Page 14 of 69

Figure 1.

External View of Vitrification Cell Shielding.

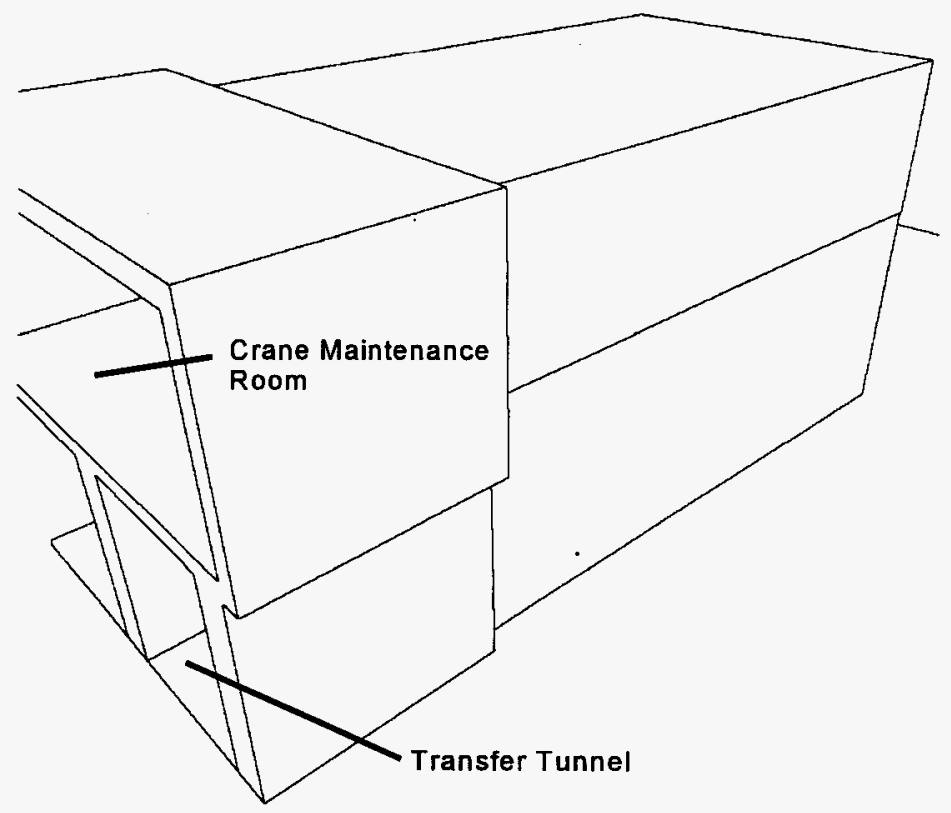


Figure 2.

Top View of Inside of Vitrification Cell.

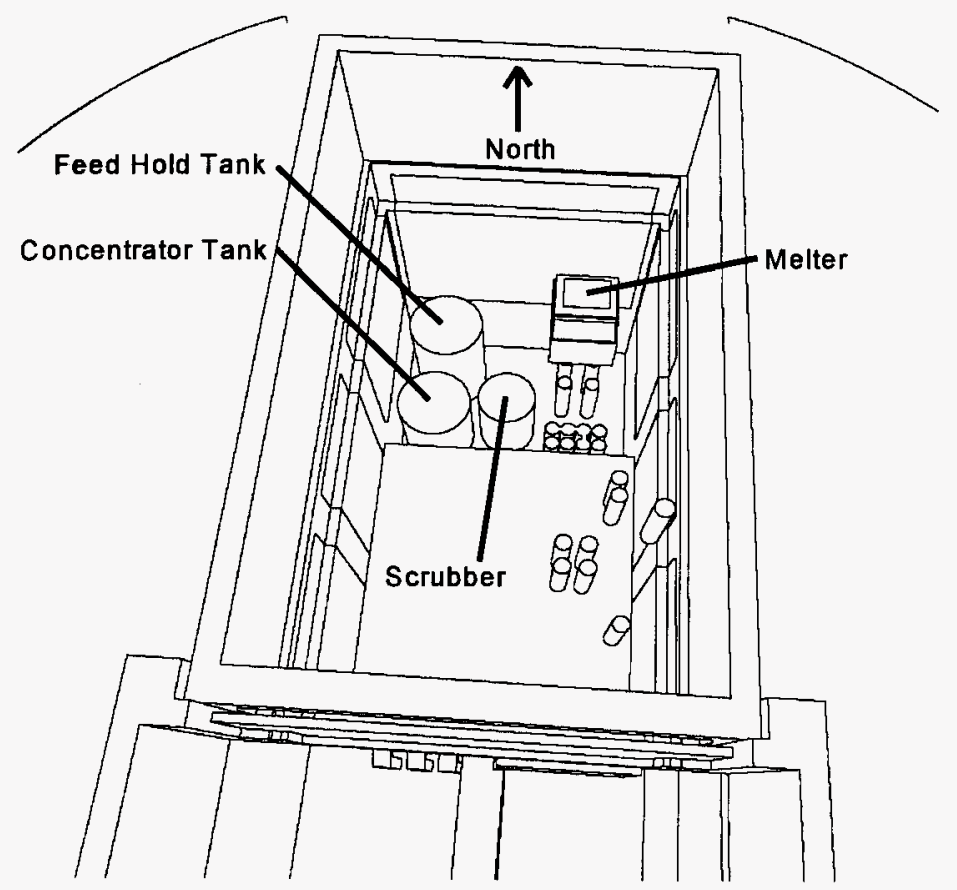




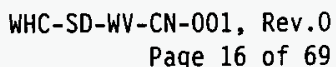

Figure 3.

Detail of Pit as Viewed from Above.

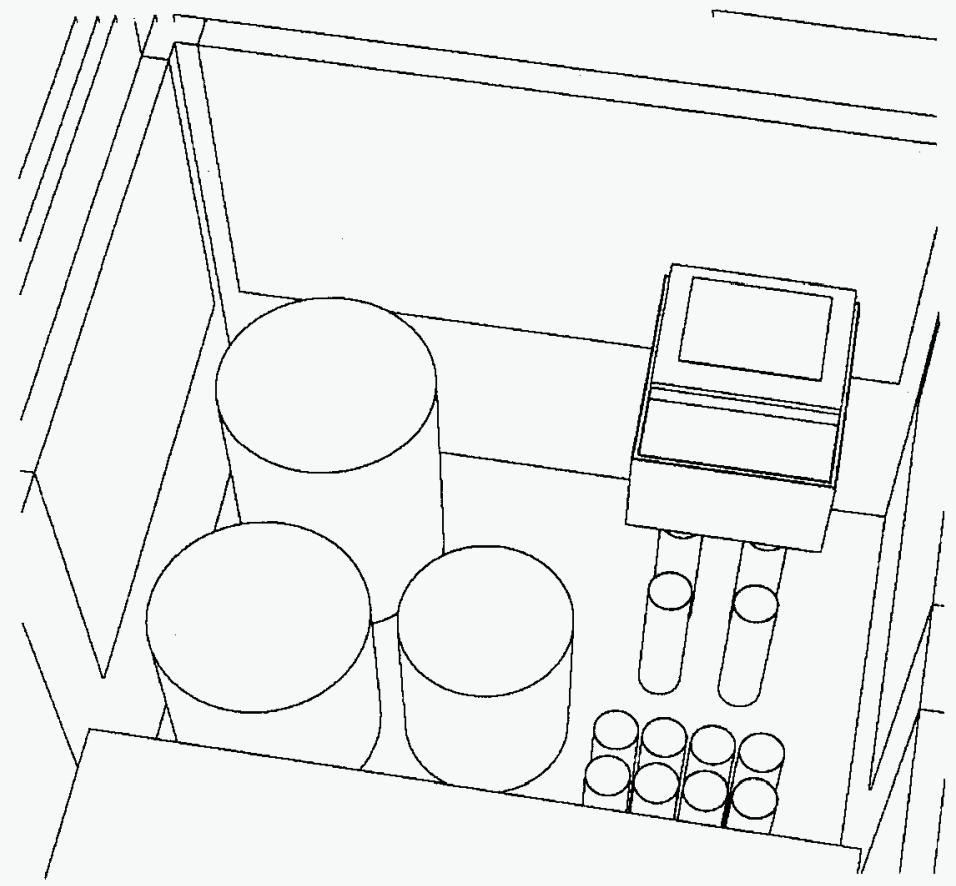


WHC-SD-WV-CN-001, Rev. 0

Page 17 of 69

Figure 4.

Cutaway of Cell as Viewed from the East.

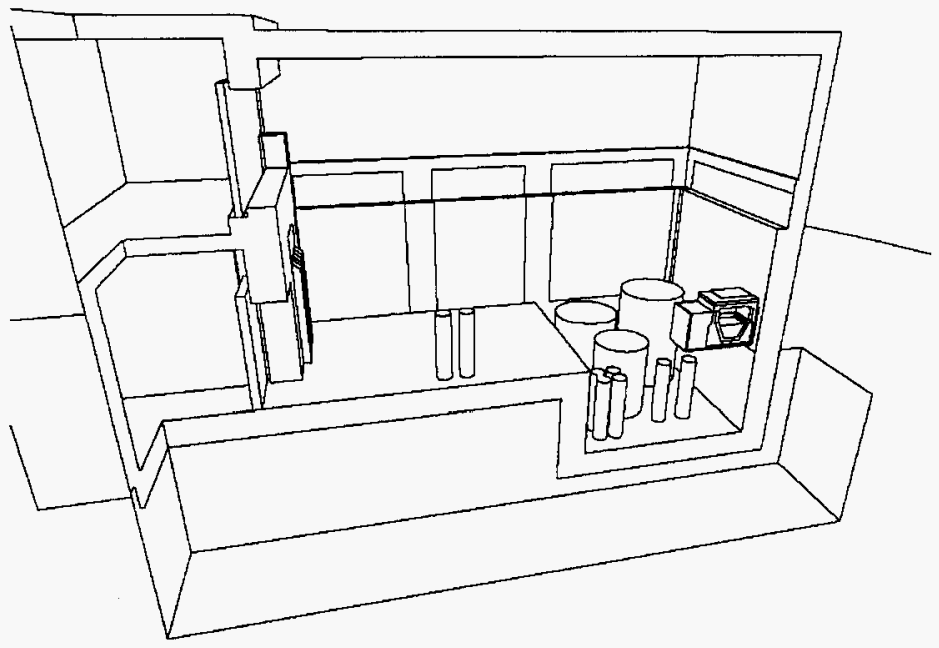


WHC-SD-WV-CN-001, Rev.0

Page 18 of 69

Figure 5.

Cutaway Detail of Melter and Pit.

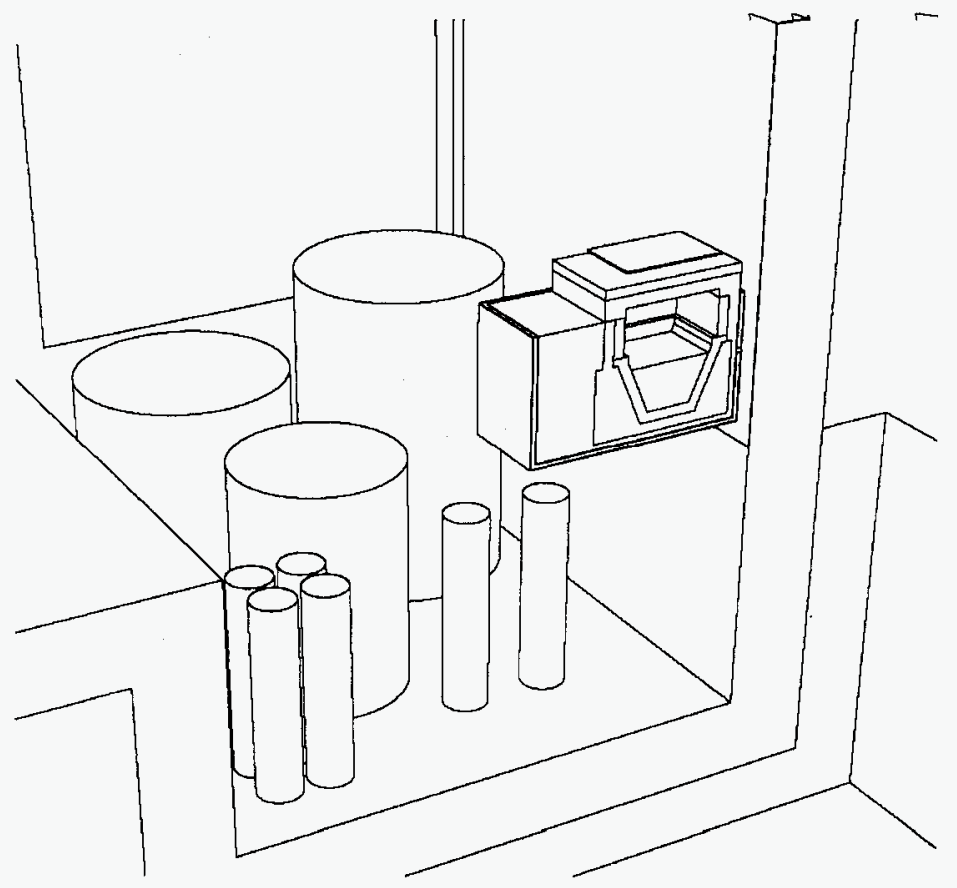


Figure 6.

Inside of Cell as Viewed from the East.

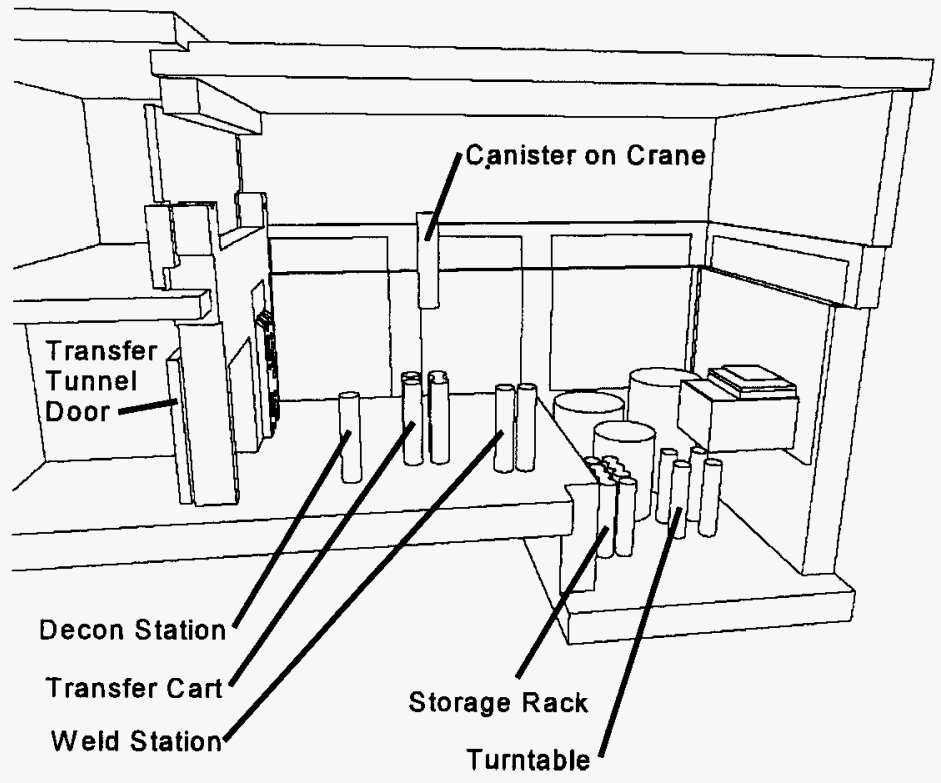


Figure 7.

Inside of Cell as Viewed from the West.

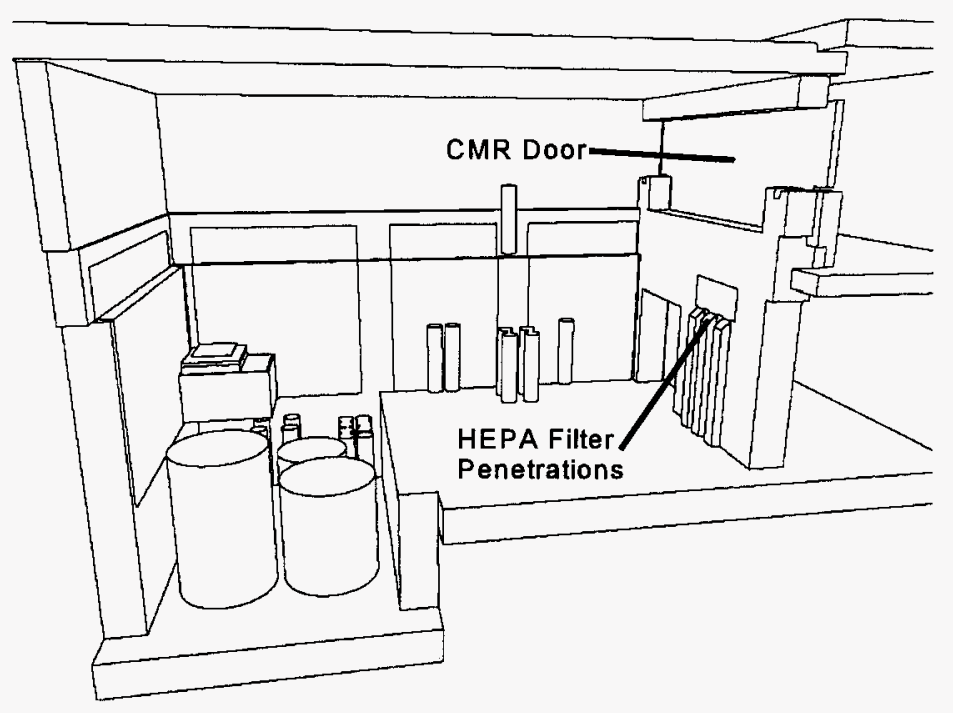




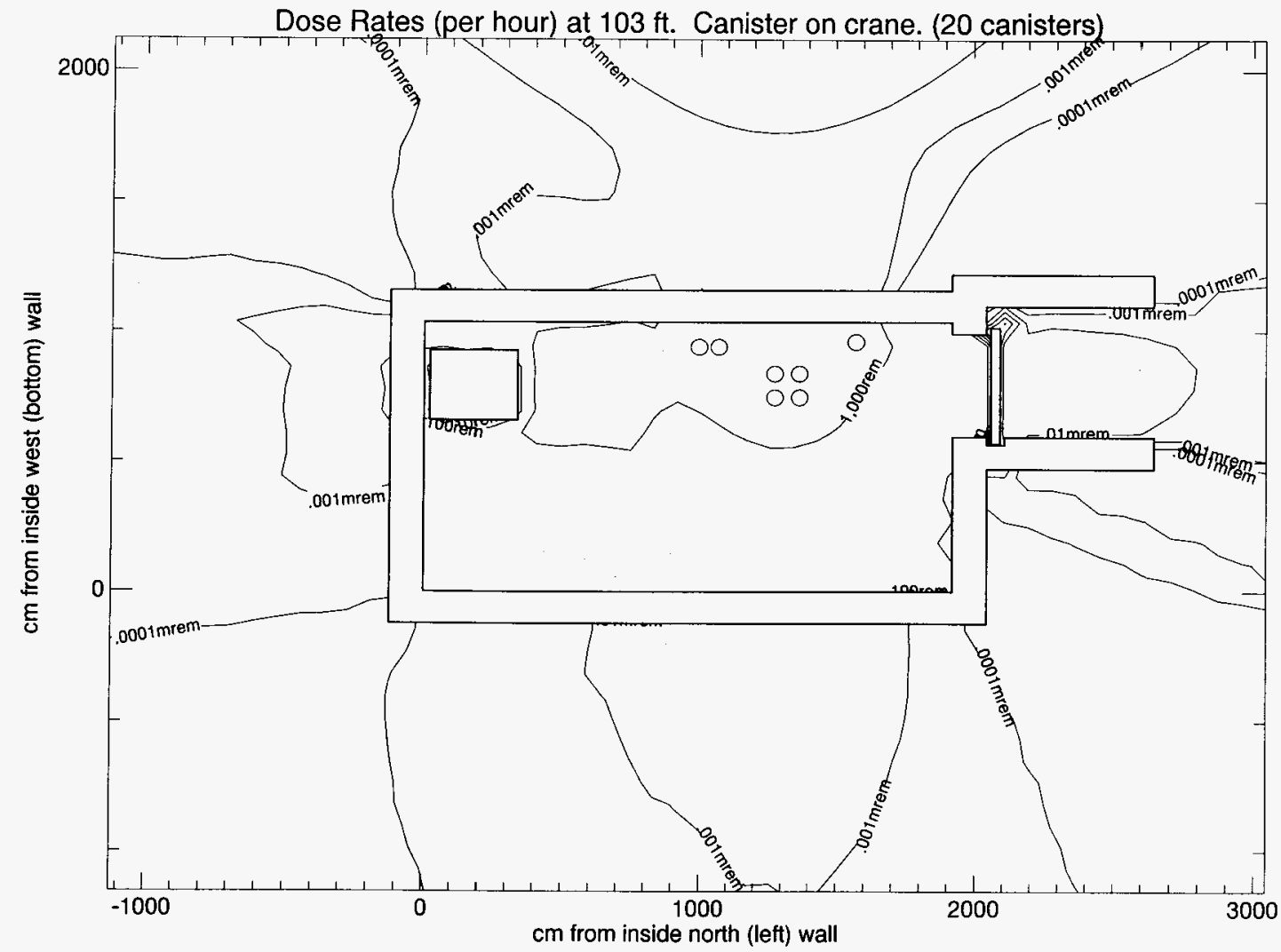

\begin{tabular}{l}
3 \\
\multirow{5}{5}{} \\
0
\end{tabular}

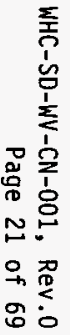




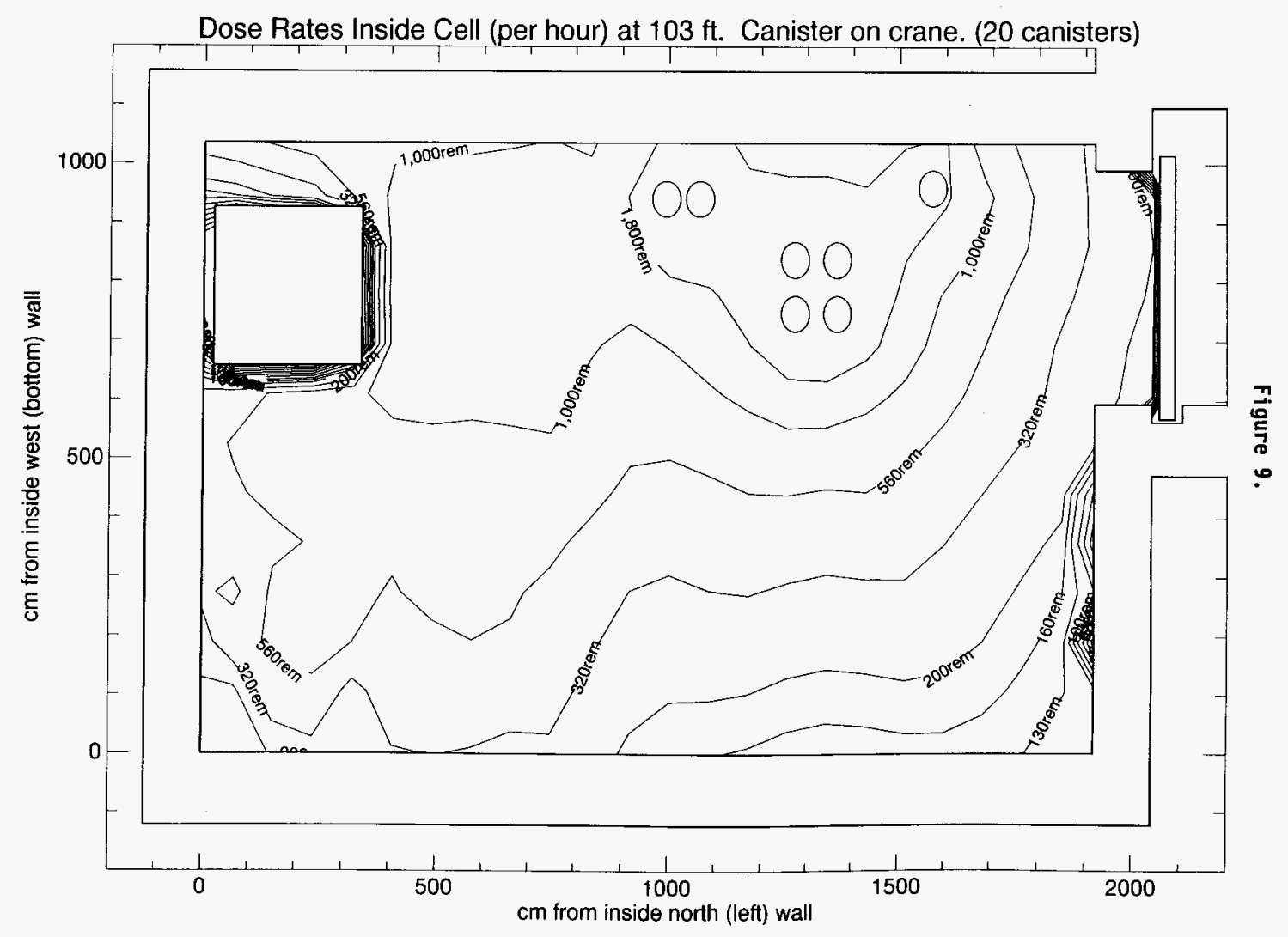

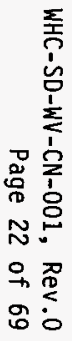




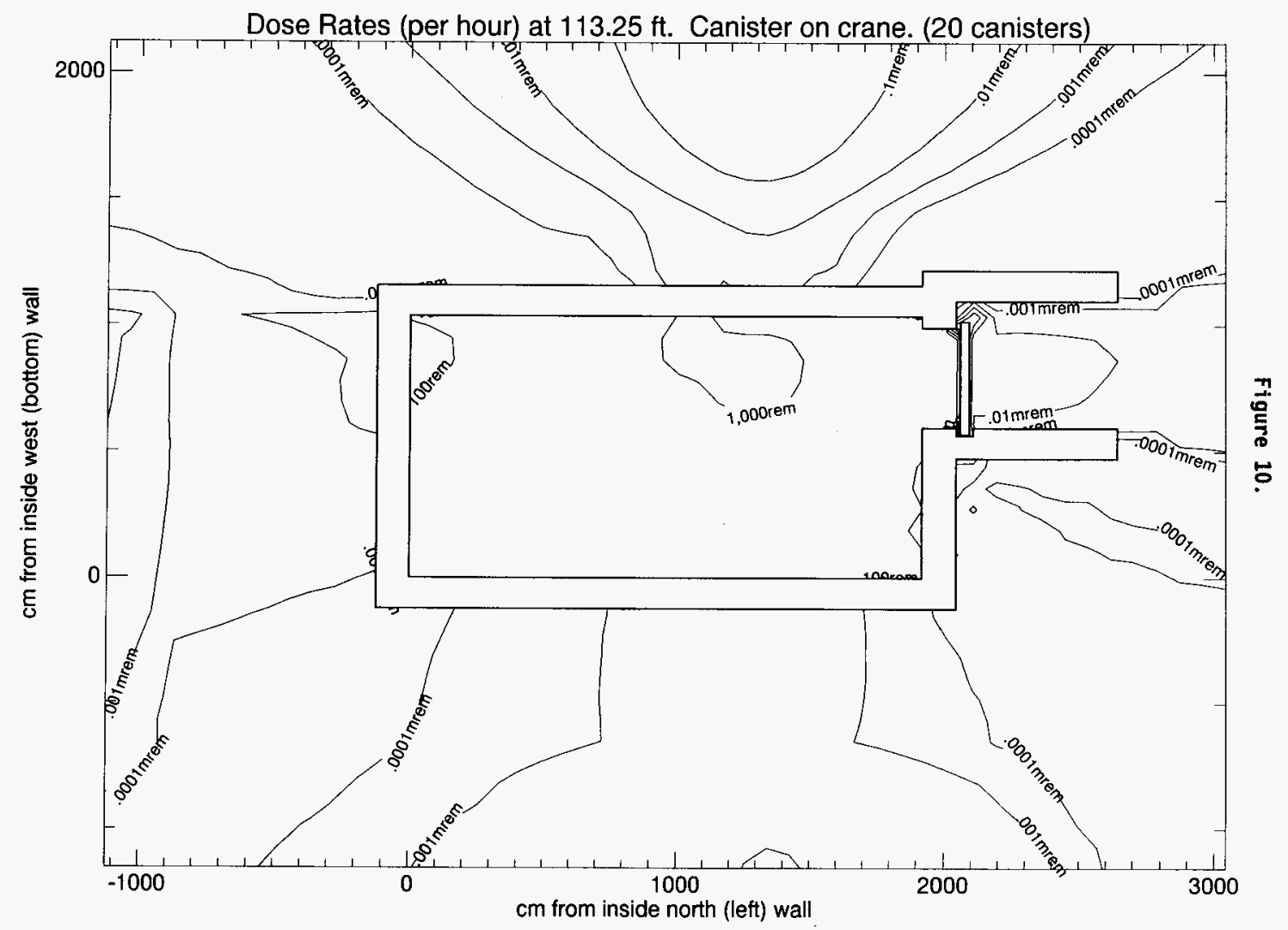

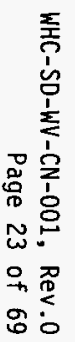


Figure 11.

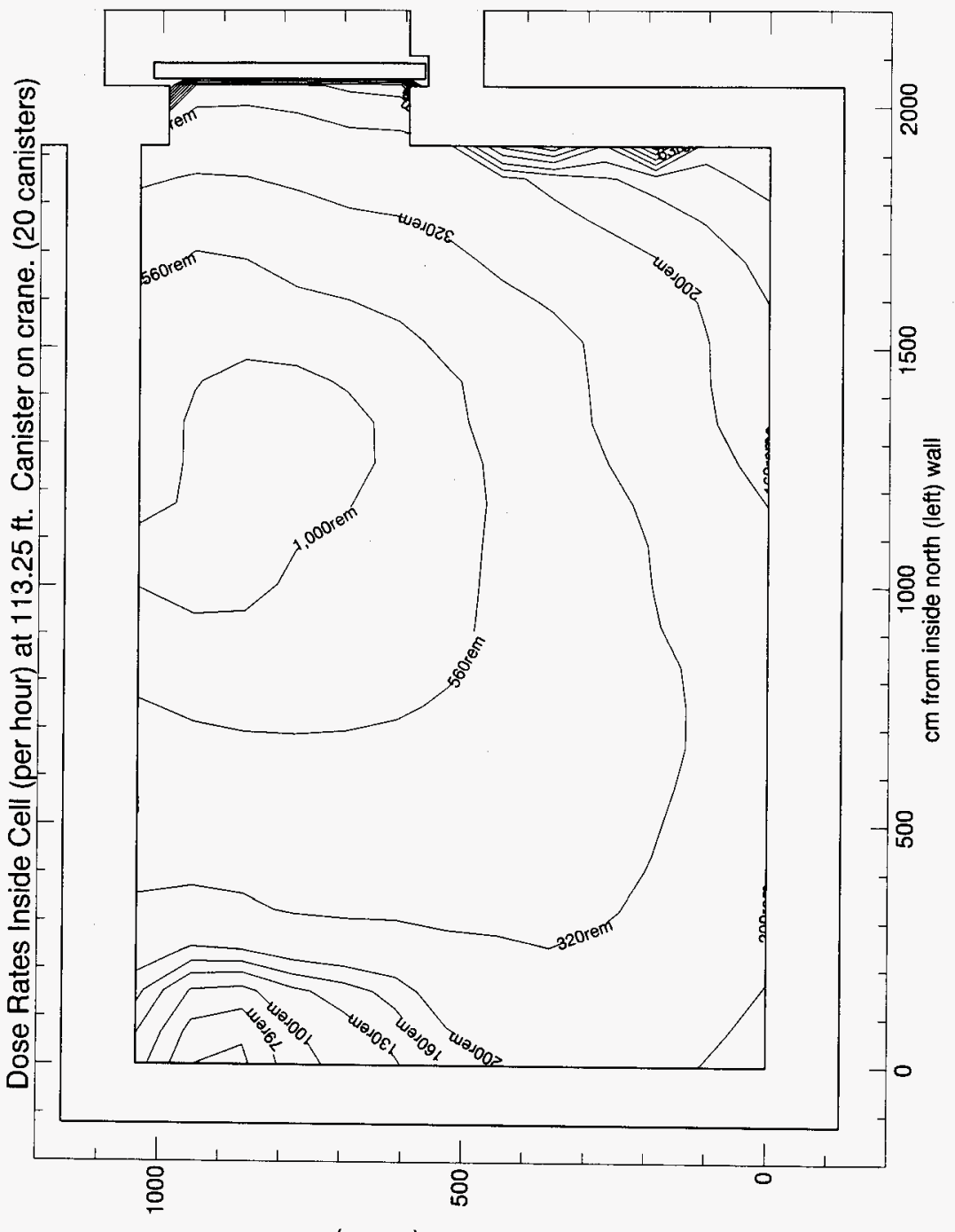

\|EM (woHOq) łsaM әp!su! woł wo 
Figure 12.

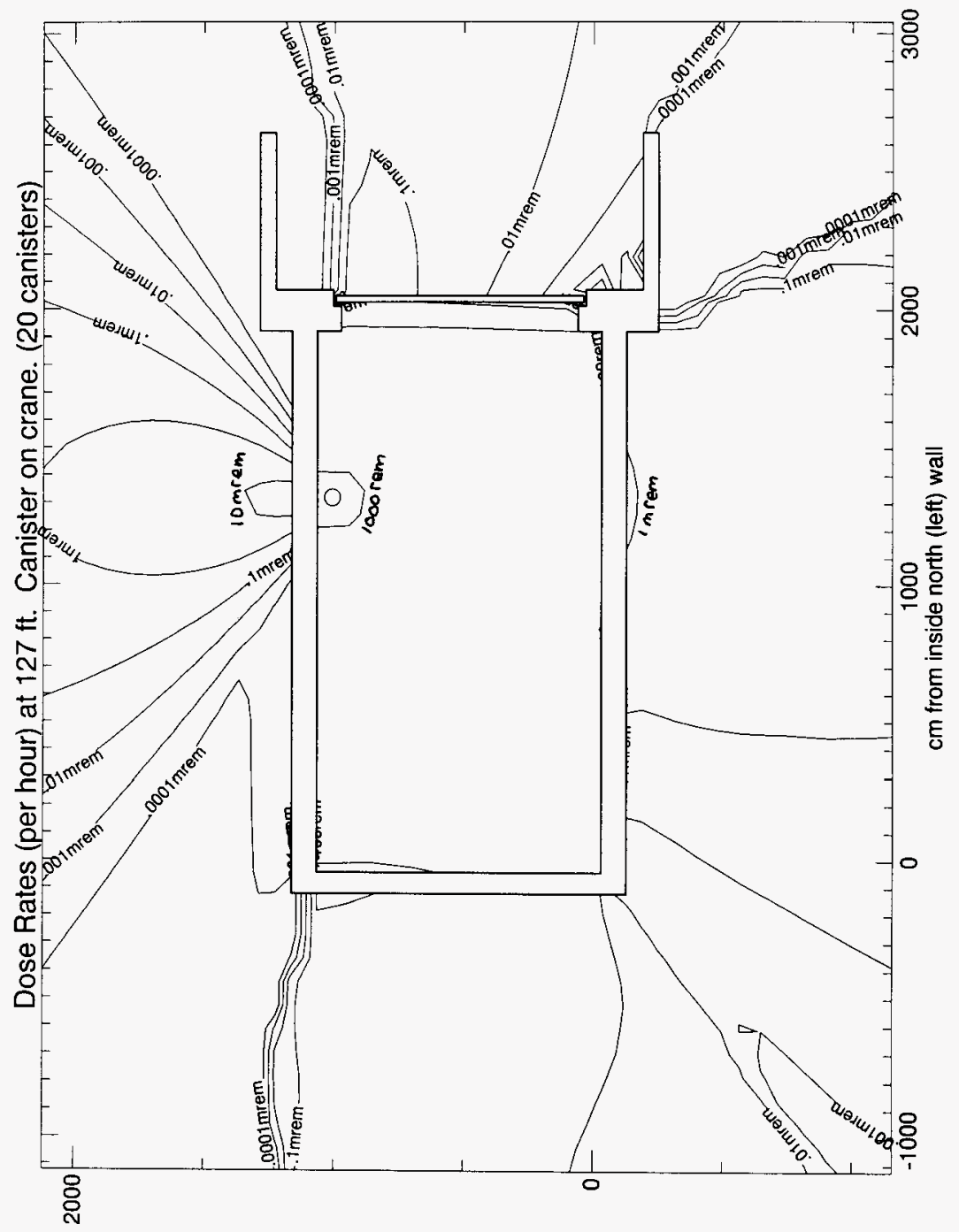

॥еM (wоноq) łsam әp!su! wод سо 
Figure 13.

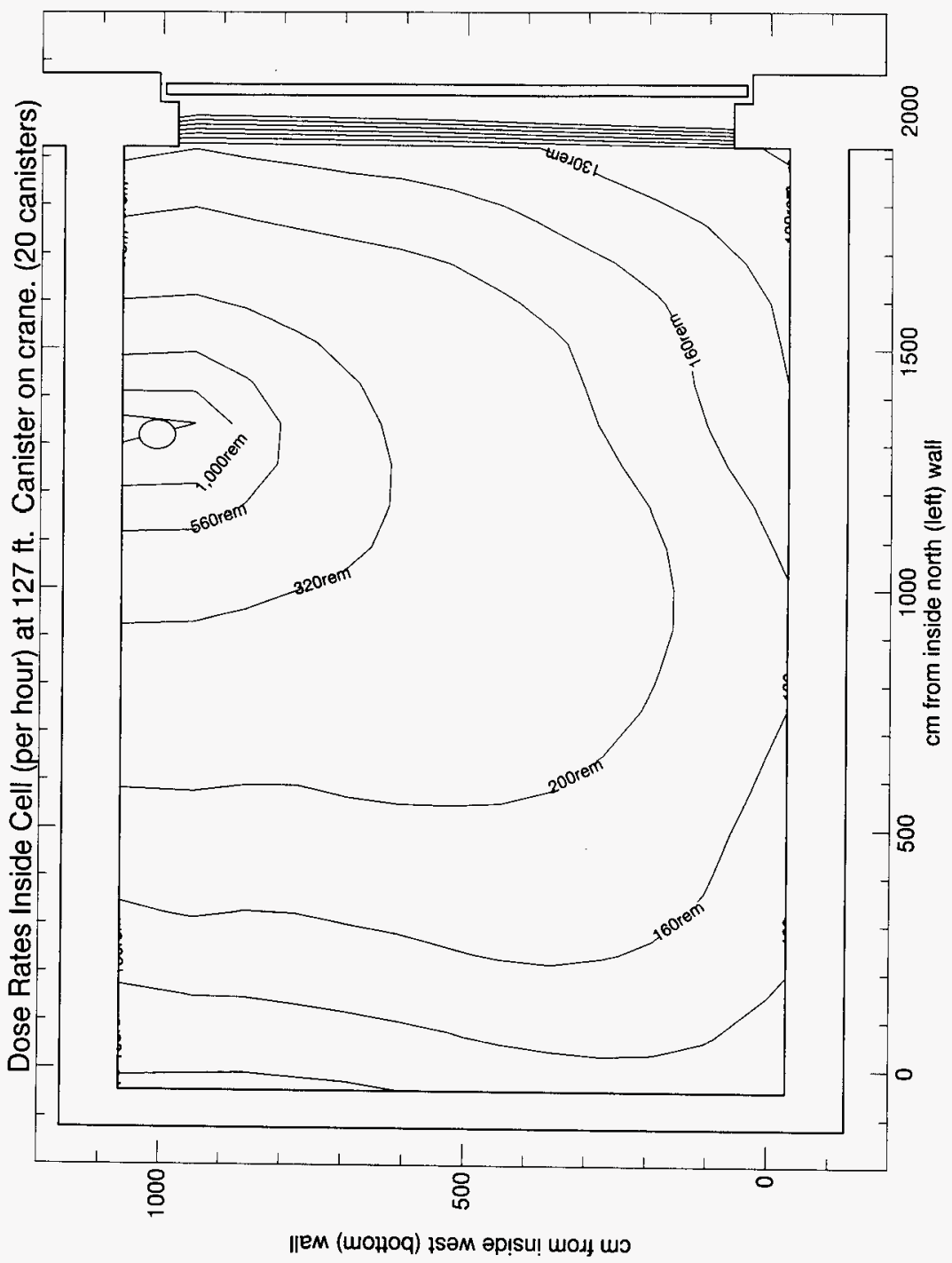




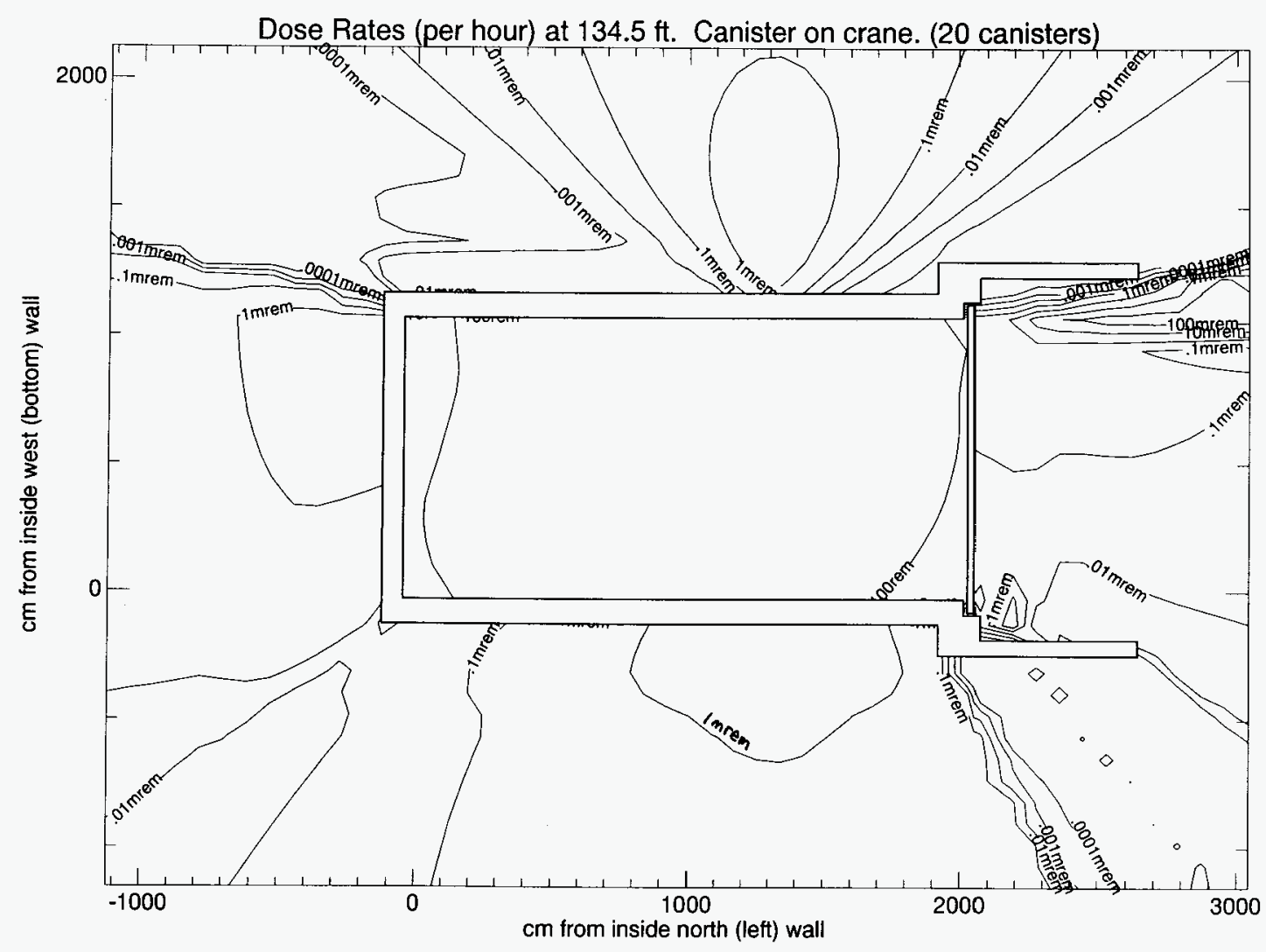

3
뭉
5
5
5

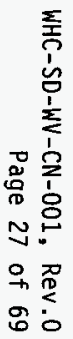


Figure 15.

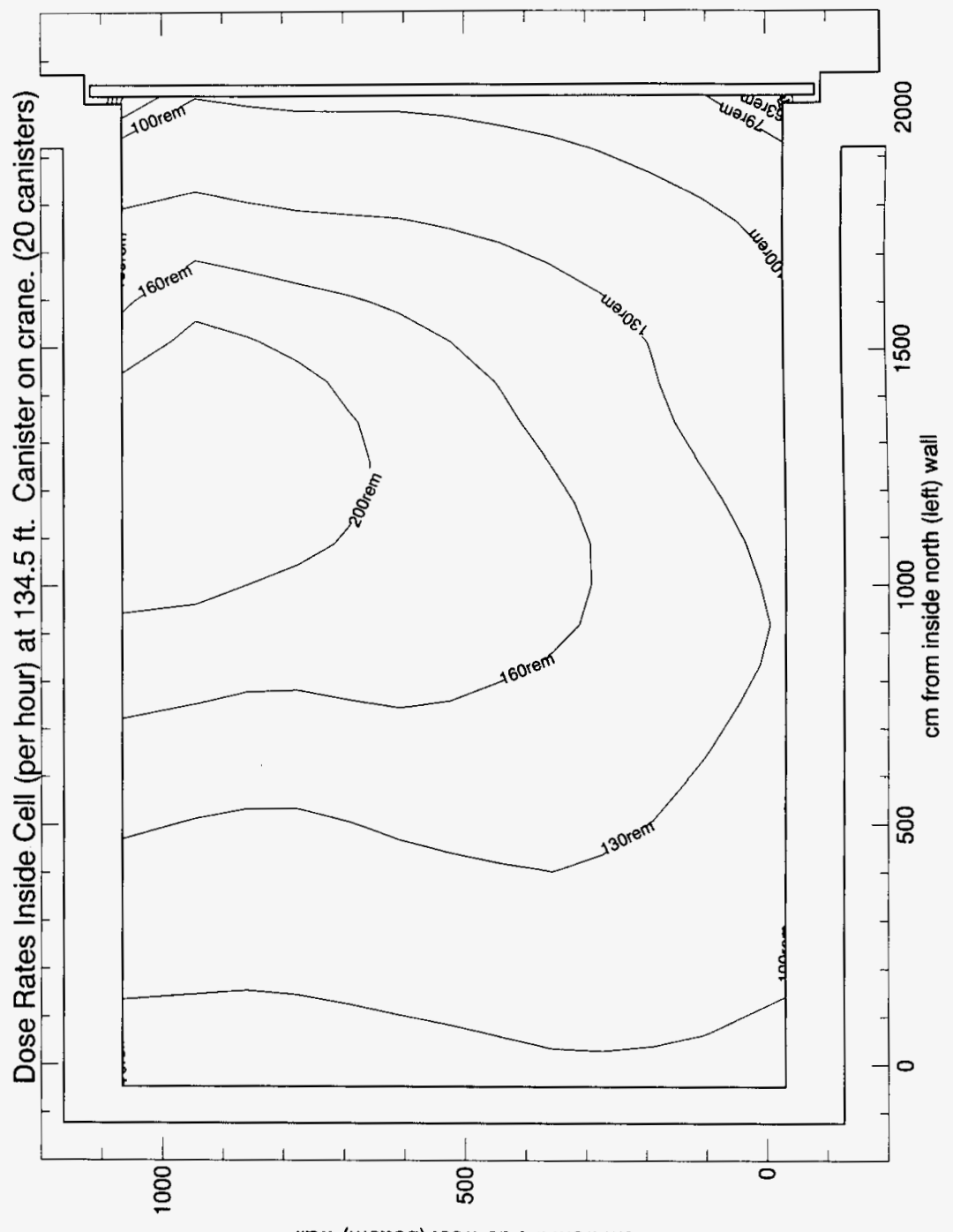

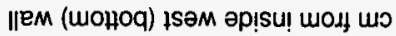




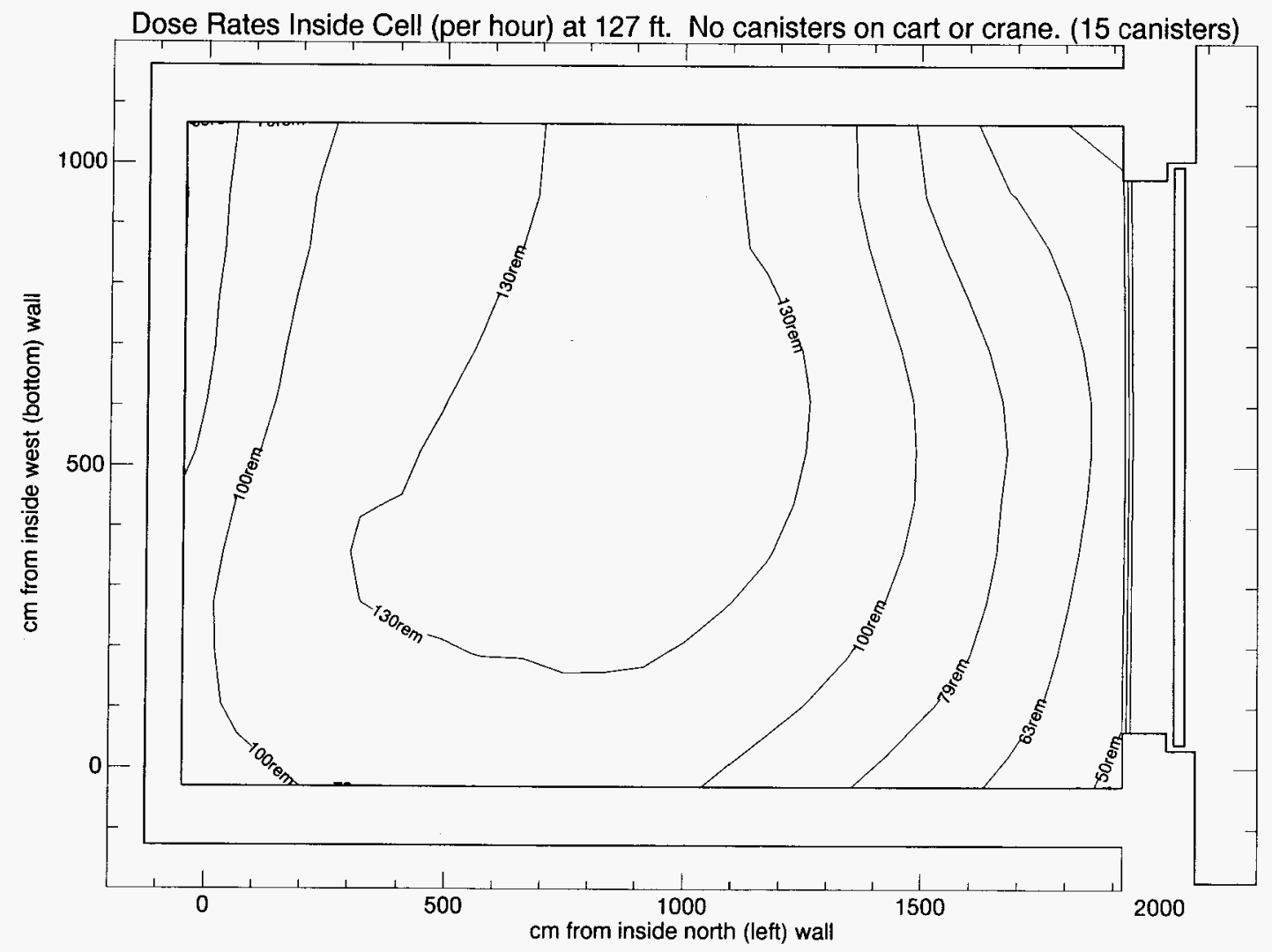

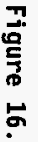

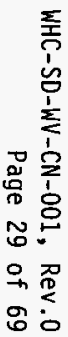


Figure 17.

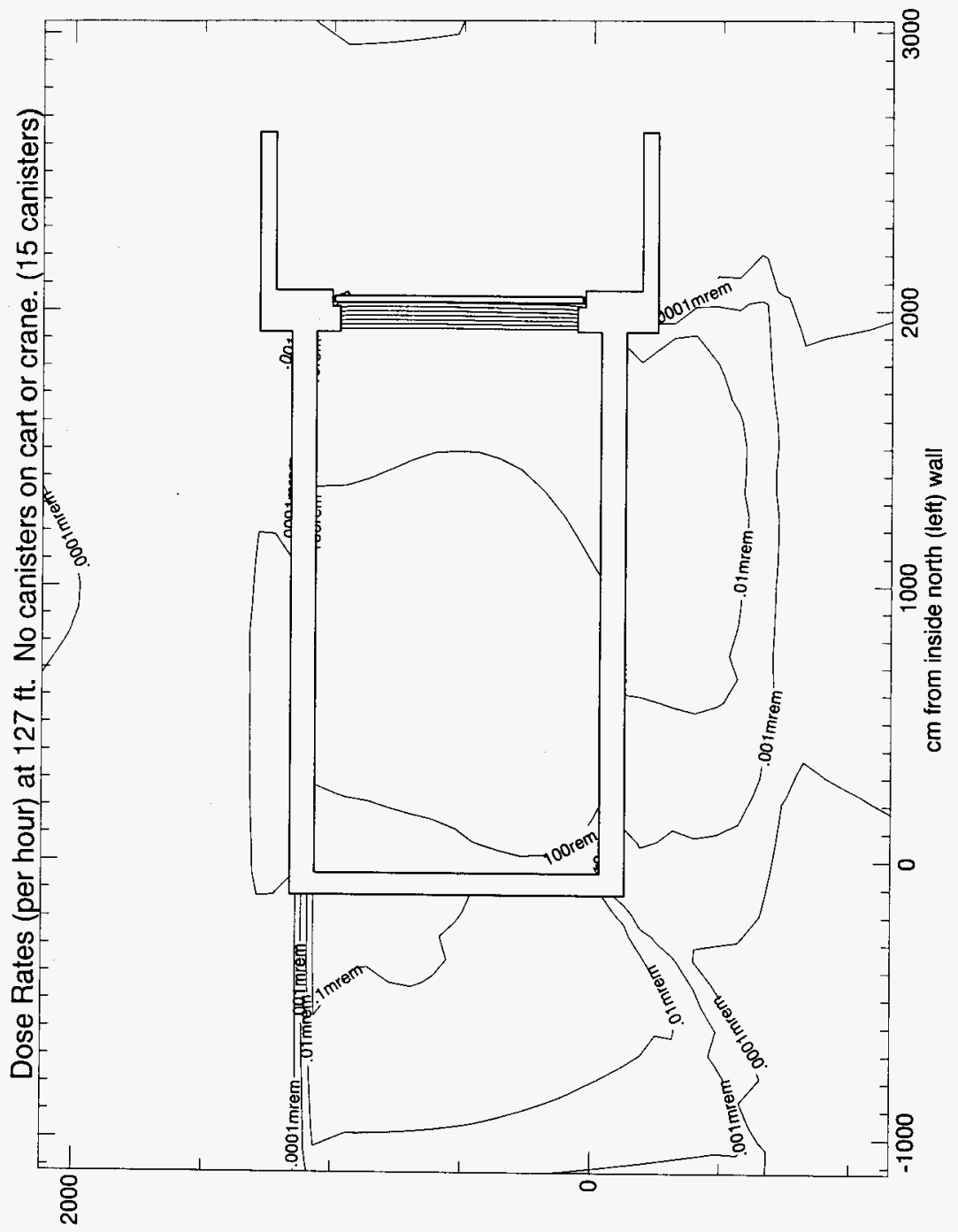

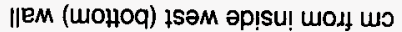


WHC-SD-WV-CN-001, Rev. 0

Page 31 of 69

Figure 18.

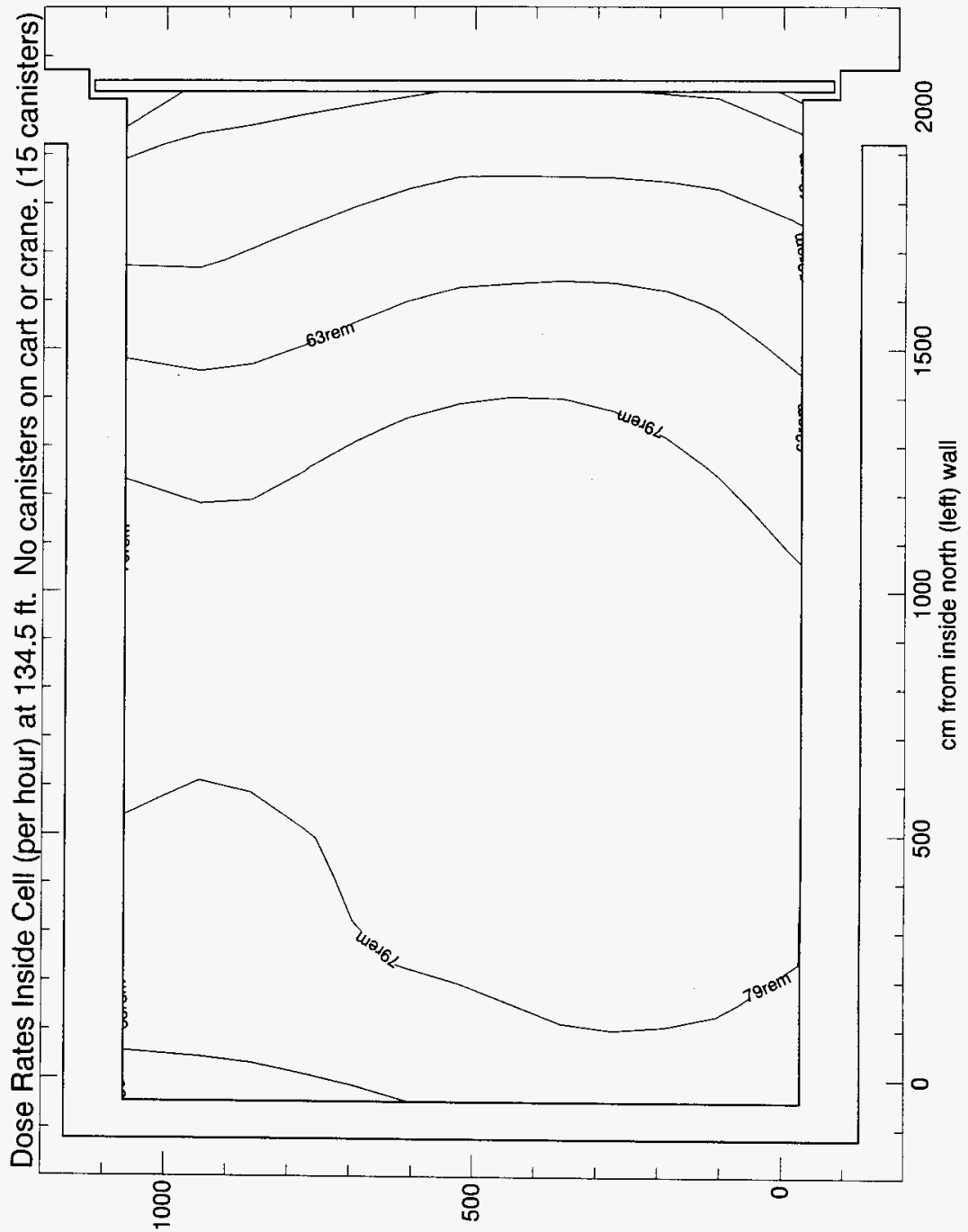

॥EM (uOHOq) tSəM әр!su! woגł سо 


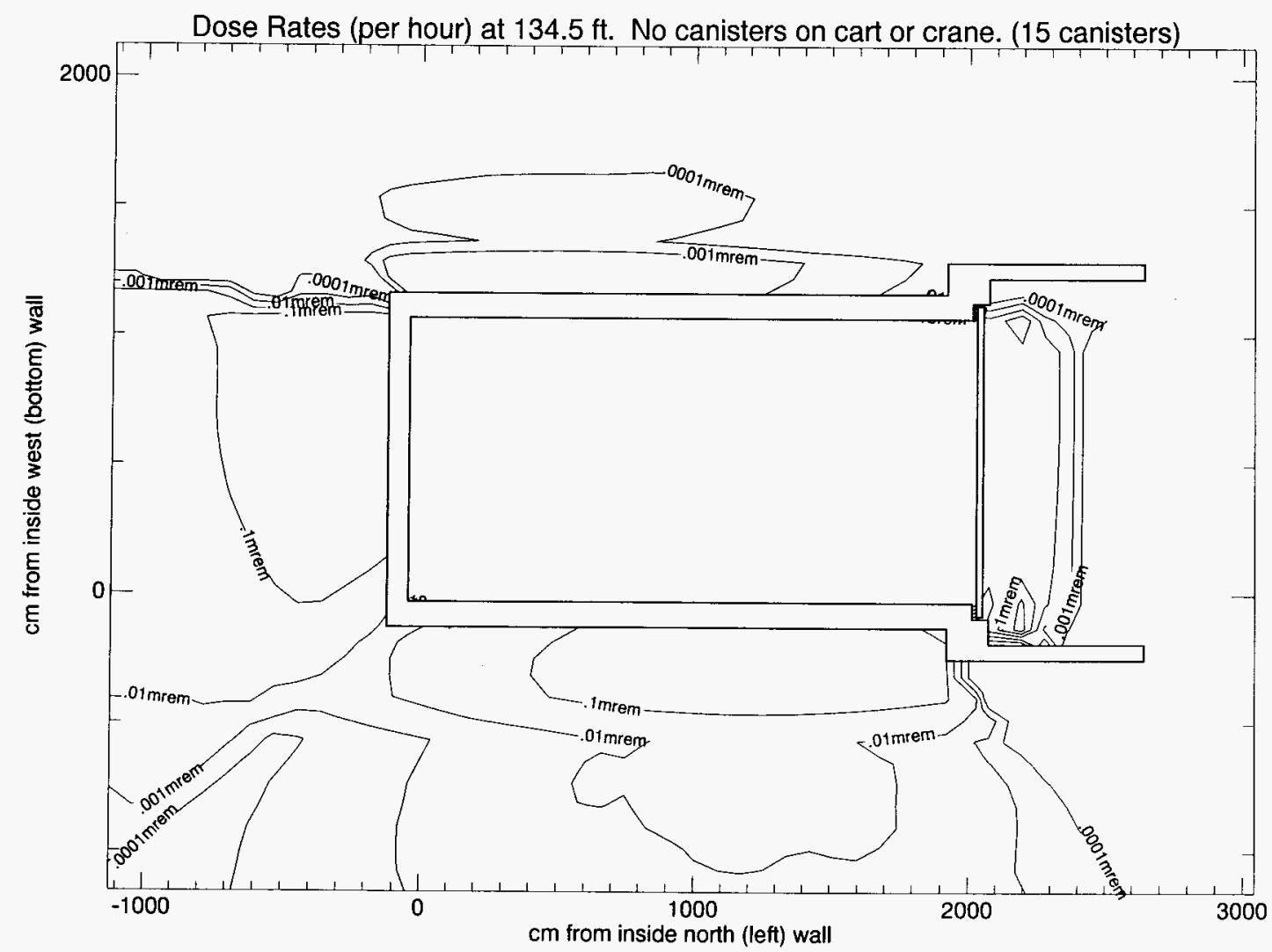

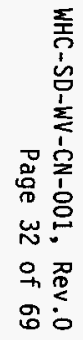




\section{Attachment A.}

CHECKLIST FOR INDEPENDENT TECHNICAL REVIEW

DOCUMENT REVIEWED_MCNP Calculational Models of West Valley Vitrification

\section{Cell and Point-Kernel Dose Contour Plots}

AUTHOR $(s)$ Victor E. Roetman

I. Method(s) of Review

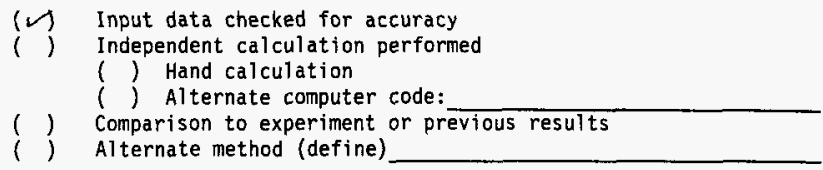

II. Checklist (either check or enter NA if not applied)

(-) Task completely defined

(-) Activity consistent with task specification

(-) Necessary assumptions explicitly stated and supported

(-) Resources properly identified and referenced

( $\rightarrow$ Resource documentation appropriate for this application

(A) Input data explicitly stated

(-) Input data verified to be consistent with original source

(-) Geometric model adequate representation of actual geometry

(-) Material properties appropriate and reasonable

(NA) Mathematical derivations checked including dimensional consistency

(NA) Hand calculations checked for errors

(-) Assumptions explicitly stated and justified

(-) Computer software appropriate for task and used within range of validity

WA) Use of resource outside range of established validity is justified

$(-)$ Software runstreams correct and consistent with results

(-) Software output consistent with input

$\left.W_{A}\right)$ Results consistent with applicable previous experimental or analytical findings

(7) Results and conclusions address all points and are consistent with task requirements and/or established limits or criteria

$(\rightarrow$ Conclusions consistent with analytical results and established limits

(-) Uncertainty assessment appropriate and reasonable

N.t) Other (define)

III. Comments:

\section{IV.}

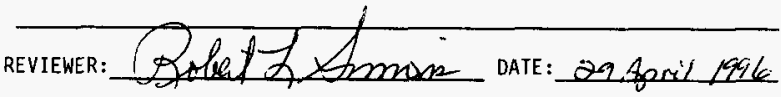


WHC-SD-WV-CN-001, Rev. 0 Attachment B

Page 34 of 69

Attachment B. MCNP Input Files 


\section{Input file for the full canister configuration (20 canisters):}

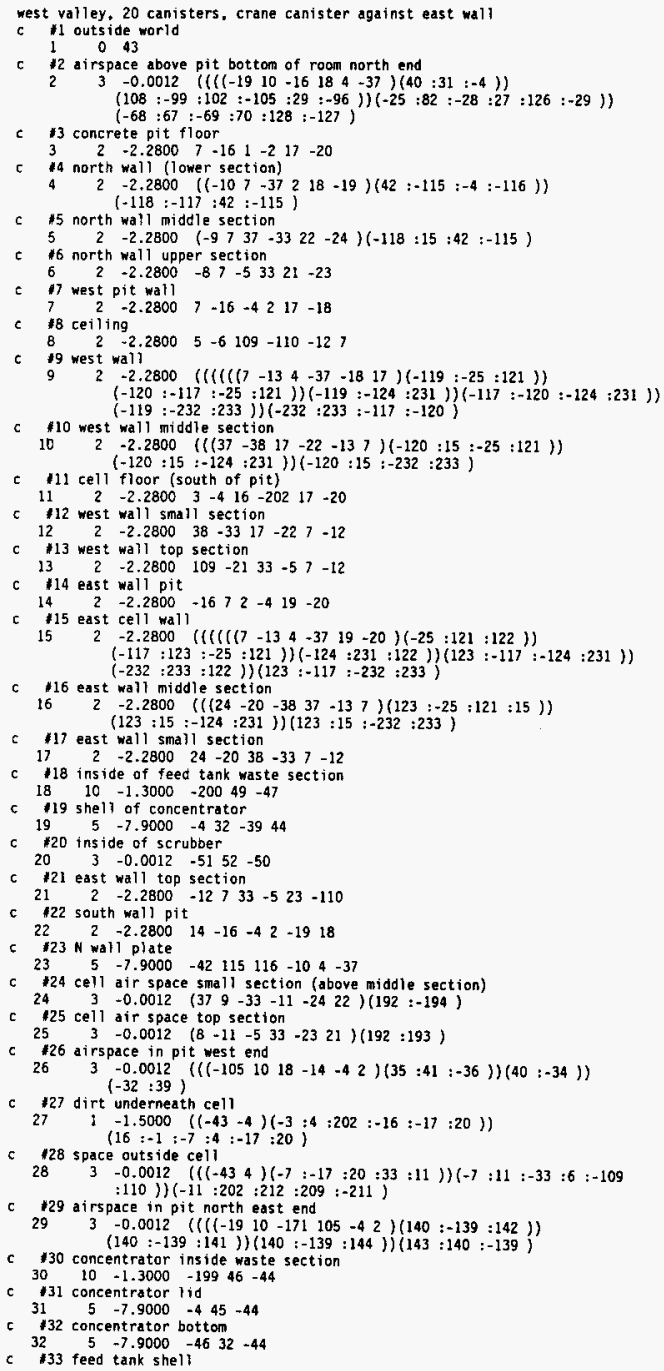


WHC-SD-WV-CN-001, Rev.0

Attachment $B$

Page 36 of 69

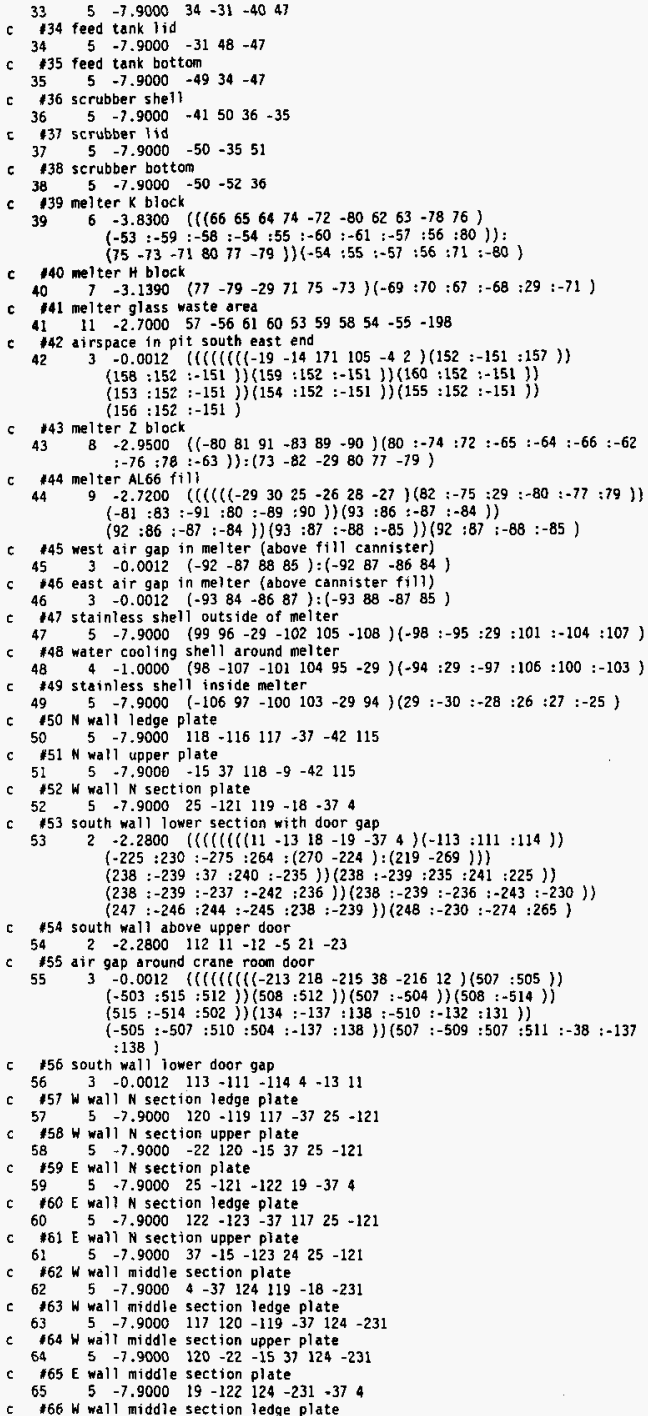


$\begin{array}{lllllllll}66 & 5 & -7.9000 & 122 & -123 & -37 & 117 & 124 & -23\end{array}$

c. $\$ 67$ melter 1 id AL-66

$67 \quad 9 \quad-2.7200\left(\begin{array}{lllllll}-201 & 29 & 28 & -27 & -82 & 25\end{array}\right)\left(\begin{array}{ll}-68 & : 67: 125:-69: 70)\end{array}\right.$

c $\$ 68$ steel plate above melter lid

$68 \quad 5 \quad-7.9000 \quad 68-67 \quad 69-70-128 \quad 127$

c $\$ 69$ south wall middle section

$692-2.2800((\{37-38 \quad 22-24-1311)(-225:-275: 270))$

$(-225:-224: 219))(-225:-269: 264)$

c 70 south wall upper section

$\begin{array}{lllllllll}70 & 2 & -2.2800 & 38 & -33 & 22 & -24 & 11 & -12\end{array}$

c 11 wall middle section upper plate

$\begin{array}{lllllllll}71 & 5 & -7.9000 & 37 & -15 & 24 & -123 & 124 & -231\end{array}$

c $\$ 72$ lower steel door

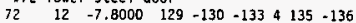

c 173 upper steel door

$73 \quad 12-7.8000((((-13438132-131-138137)(510: 505))(-504: 510))$ $(507: 509))(507:-511)$

174 turntable cannister shell

$745-7.9000 \quad 145-141-140139$

c 175 turntable cannister she 11

$75 \quad 5 \quad-7.9000 \quad 147-144 \quad-140139$

c $\quad 76$ turntable cannister shel1

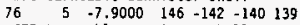

c $\$ 77$ turntable cannister shell

I7 $5 \quad 5 \quad-7.9000 \quad-143 \quad 148 \quad-140 \quad 139$

78 turntable cannister inside

$78 \quad[1 \quad-2.7000-145-150149$

c $\$ 79$ turntable cannister inside

$79 \quad 11-2.7000-147-150149$

c $\$ 80$ turntable cannister inside

$8011-2.7000-146-150149$

c $\quad 81$ turntable cannister inside

$81 \quad 11-2.7000-148-150149$

c $\quad 82$ turntable cannister lid

$82 \quad 3-0.0012-145150-140$

c 183 turntable cannister botton

$8355-7.9000-145-149 \quad 139$

- 84 turntable cannister lid

$843-0.0012-147$ 150-140

c 85 turntable cannister bottom

$\begin{array}{llllll}85 & 5 & -7.9000 & -149 & 139 & -147\end{array}$

c $\quad 86$ turntable cannister 1 id

$\begin{array}{llllll}86 & 3 & -0.0012 & -146 & 150 & -140\end{array}$

187 turntable cannister botton
$87 \quad 5-7.9000-146-149139$

6 $\$ 88$ turntable cannister lid

$\begin{array}{llllll}88 & 3 & -0.0012 & -148 & 150 & -140\end{array}$

c 189 turntable cannister bottorn

$89 \quad 5 \quad-7.9000-149 \quad 139-148$

c $\quad 190$ cable passageway

$903-0.0012 \quad 505-503\left(\left((11-516517-521):\left(\begin{array}{lllll}-516 & -520 & 521 & 519\end{array}\right)\right)=\right.$ $(-518519-215520))$

c 19] rack cannister inside

$9111-2.7000-169170-164$

c 92 rack cannister inside

$92 \quad 11-2.7000-169 \quad 170-163$

c 93 rack cannister inside

$93 \quad 11-2.7000 \quad-169170-162$

c 194 rack cannister inside

$94 \quad 11 \quad-2,7000 \quad-169 \quad 170-161$

c $\$ 95$ rack cannister inside

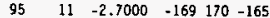

c $\$ 96$ rack cannister inside

$9611-2.7000=169170-166$

c $\$ 97$ rack cannister inside

$\begin{array}{llllll}97 & 11 & -2.7000 & -169 & -167 & 170\end{array}$

C 998 rack cannister inside

$98 \quad 11-2.7000-169 \quad 170-168$

c 99 washer cannister botton

$995-7.9000180-190-188$

c 100 cable passageway

$1003-0.0012-504502\left(\left((-51651711-521):\left(\begin{array}{llllll}-516 & -520 & 521 & 519))\end{array}\right.\right.\right.$ $(-518519-216520)$

c 101 rack cannister shell

$10155-7.9000-152151 \quad 164-156$

c 102 rack cannister shell

$\begin{array}{llllllll}102 & 5 & -7.9000 & -152 & 151 & 163 & -155\end{array}$

c 103 rack cannister shell

$\begin{array}{llllllll}103 & 5 & -7.9000 & -152 & 151 & 162 & -154\end{array}$

c 104 rack cannister shell

$\begin{array}{llllllll}104 & 5 & -7.9000 & -152 & 151 & 161 & -153\end{array}$

c 105 rack cannister shell 
$\begin{array}{lllllll}105 & 5 & -7.9000 & -152 & 151 & 168 & -160\end{array}$ c 106 rack cannister shell

$\begin{array}{lllllll}106 & 5 & -7.9000 & -152 & 151 & 167 & -159\end{array}$

c 107 rack cannister shell

$107 \quad 5 \quad-7.9000 \quad-152 \quad 151 \quad 166-158$

c tlos rack cannister shell

$\begin{array}{llllllll}108 & 5 & -7.9000 & -152 & 151 & 165 & -157\end{array}$

c 109 rack cannister lid

$1093 \begin{array}{lllll}160 & -0.0012 & -164 & 169 & -152\end{array}$

c $\$ 110$ rack cannister bottom

$\begin{array}{llllll}110 & 5 & -7.9000 & -164 & -170 & 151\end{array}$

c 1111 rack cannister 1 id

$11133-0.0012-163 \quad 169-152$

c 1112 rack cannister bottom

$11255-7.9000-163-170 \quad 151$

c $\$ 113$ rack cammister lid

$213 \quad 3 \quad-0.0012-162 \quad 169-152$

c $\$ 114$ rack cannister bottom

$\begin{array}{llllll}114 & 5 & -7.9000 & -162 & 151 & -170\end{array}$

c \$115 rack cannister idd

$\begin{array}{llllll}115 & 3 & -0.0012 & -161 & 169 & -152\end{array}$

c 116 rack cannister bottom

$11655-7.9000-161-170151$

c 1117 rack cannister 1 id

$117 \quad 3-0.0012-168169-152$

c $\quad$ t118 rack cannister bottom

$118 \quad 5-7.9000-170 \quad 151-168$

c $\$ 119$ rack cannister lid

$\begin{array}{llllll}119 & 3 & -0.0012 & -167 & 169 & -152\end{array}$

c $\$ 120$ rack cannister bottom

$\begin{array}{llllll}120 & 5 & -7.9000 & -167 & -170 & 151\end{array}$

c 121 rack cannister lid

$1213=0.0012 \quad 169-152-166$

c \#122 rack cannister bottom

$122 \quad 5 \quad-7.9000-166 \quad 151-170$

c 123 rack cannister lid

$\begin{array}{llllll}123 & 3 & -0.0012 & -165 & 169 & -152\end{array}$

c 124 rack cannister bottom

$1245-7.9000-165-170151$

c 125 airspace lower section south end

$1253-0.0012$ ( ( ( ( ( ( ( (16 -11 $18-19-374)(-180: 181: 172))$

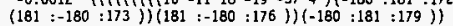

$(181:-180: 178)\}(181:-180: 177))(181:-180: 174)))$ $(-226:-250:-274: 265:(271-223):(220-268))$

c 1126 welder canaister inside

$126 \quad 11-2.7000-191190-183$

c $\quad 127$ welder cannister inside

$127 \quad 11-2.7000-191190-182$

c 128 cart cannister inside

$\begin{array}{llllll}128 & 11 & -2.7000 & -191 & 190 & -184\end{array}$

c $\quad 129$ cart cannister inside

$129 \quad 11-2.7000-191 \quad 190-185$

c 130 cart cannister inside

$13011-2.7000-191 \quad 190-186$

c 1131 cart cannister inside

$\begin{array}{llllll}131 & 11 & -2.7000 & -191 & 190 & -187\end{array}$

$6 \quad 132$ washer cannister inside

$132 \quad 11-2.7000-191190-188$

c 133 extra wall section $S$ wall

$1332-2.2800230-248114271-223$

c 134 welder cannister shell

$\begin{array}{lllllll}134 & 5 & -7.9000 & -181 & 180 & 182 & -172\end{array}$

c 135 welder cannister shell

$135 \quad 5 \quad-7.9000-181 \quad 180183-173$

c 136 washer cannister she11

$\begin{array}{lllllll}136 & 5 & -7.9000 & -181 & 180 & -174 & 188\end{array}$

c 137 extra wall section 5 wall

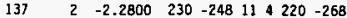

c 138 cart cannister shell

$\begin{array}{lllllll}138 & 5 & -7.9000 & -181 & 180 & 184 & -176\end{array}$

c 1139 cart cannister shell

$\begin{array}{lllllll}139 & 5 & -7.9000 & -181 & 180 & 187 & -177\end{array}$

c 140 cart cannister shell

$140 \quad 5-7.9000-181 \quad 180185-179$

c 141 cart cannister shell

$\begin{array}{llllllll}1415 & -7.9000 & -181 & 180 & 186 & -178\end{array}$

c 142 cart cannister lid

$\begin{array}{llllll}142 & 5 & -7.9000 & 191 & -181 & -185\end{array}$

C $\$ 143$ cart cannister bottom

$1435-7.9000 \quad 180-190-185$

c $\$ 144$ cart cannister lid

$144 \quad 5 \quad-7.9000 \quad-181 \quad 191 \quad-186$ 


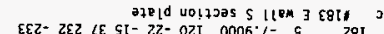

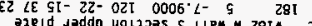

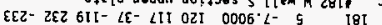

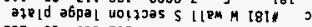
b $\angle \varepsilon-\varepsilon \varepsilon 2-8 \mathrm{I}-6 \mathrm{It} 2 \varepsilon 20005^{\circ} L^{-} \mathrm{g}$ OBI afrid uotzoas S liem $\mathrm{m}$ 08th,

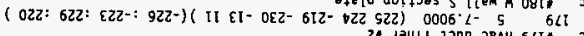

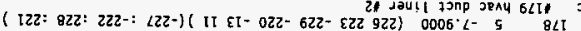

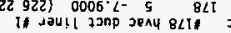

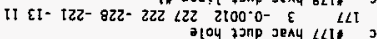
( EST: 05T: 9EI: 5EI-: $52 \mathrm{I}^{-1}$

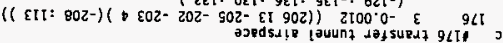

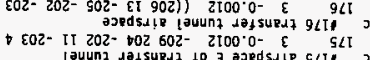

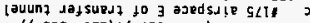

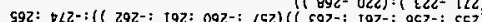

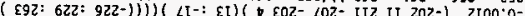

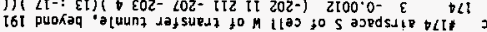

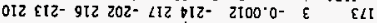

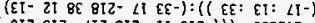

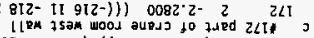
(ZT EI- BE STZ OZ- EE-): (( $\varepsilon \varepsilon: 0 Z: \varepsilon I)$

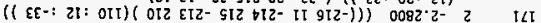

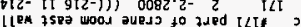

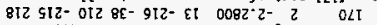

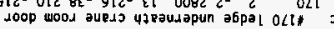
EIZ- OTZ II $202-\angle T Z-$ IIZ $0082 \cdot z-z \quad 69 t$

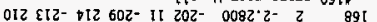

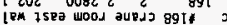

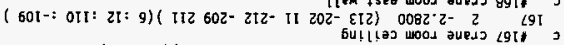
(EI: 0Z) ( + EOZ- II ZOZ1184 3 lautun dejsurdz 995

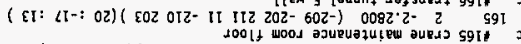

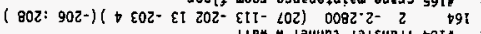
LIRM M lauunt safsurde tot

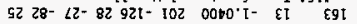

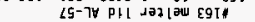
$00 z$ ct- 8t- zT00.0- E 291 $66 \mathrm{t} \mathrm{th-} \mathrm{St-} 2100^{\circ} 0^{-}$E t91

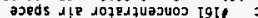

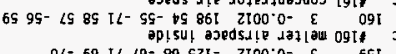

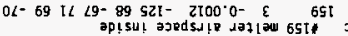
S6I- 65t C6I- $0006^{\circ} L^{-}$g 8ST

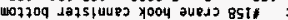

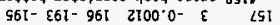

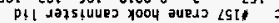
$96 T^{-56 t-\angle 6 t} 0001^{\circ} z-$ it $9 \mathrm{GL}$

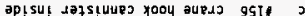
†6I $96126 I^{-} 86 I^{-} 0006^{\circ} L^{-} \quad$ S 5St Lleys dars!uues yoo4 ourdo sct* o 88I- I81- I6I 0006' $L=5$ bST p!t dazstutes jaqsen tgip. E8I- 061 I- 081 $0006^{\circ} /$ - g ECT uo7z0q da7s!utues Japłam Est: E8I- I8I- I6I ट100. E ZSL pIl dazsiuuro japlam zste $28 \mathrm{I}-06 \mathrm{~T}-08 \mathrm{I} \quad 0006^{\circ} L^{-} S$ IST woztoq dazsluups daptam TGI 28I- 18I- 161 2100.0- \& 0SI p!l da2sivurs daplä ost: L8I- 06I- 081 0006'L s 6bt

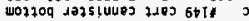

$\angle 8 I-$ I8I- I6I $0006^{\prime} L^{-}$S BDT

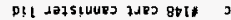

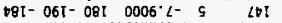

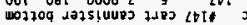
68I- I8I- I6I $0006^{\circ} \mathrm{L}^{-} 5$ 9tI p!l lazs!uues 7.185 90tí? 98I- $08 \mathrm{~T} 06 \mathrm{~T}-0006^{\circ} L^{-} \mathrm{S}$ S T T volioq atsitules 7aej soll, 


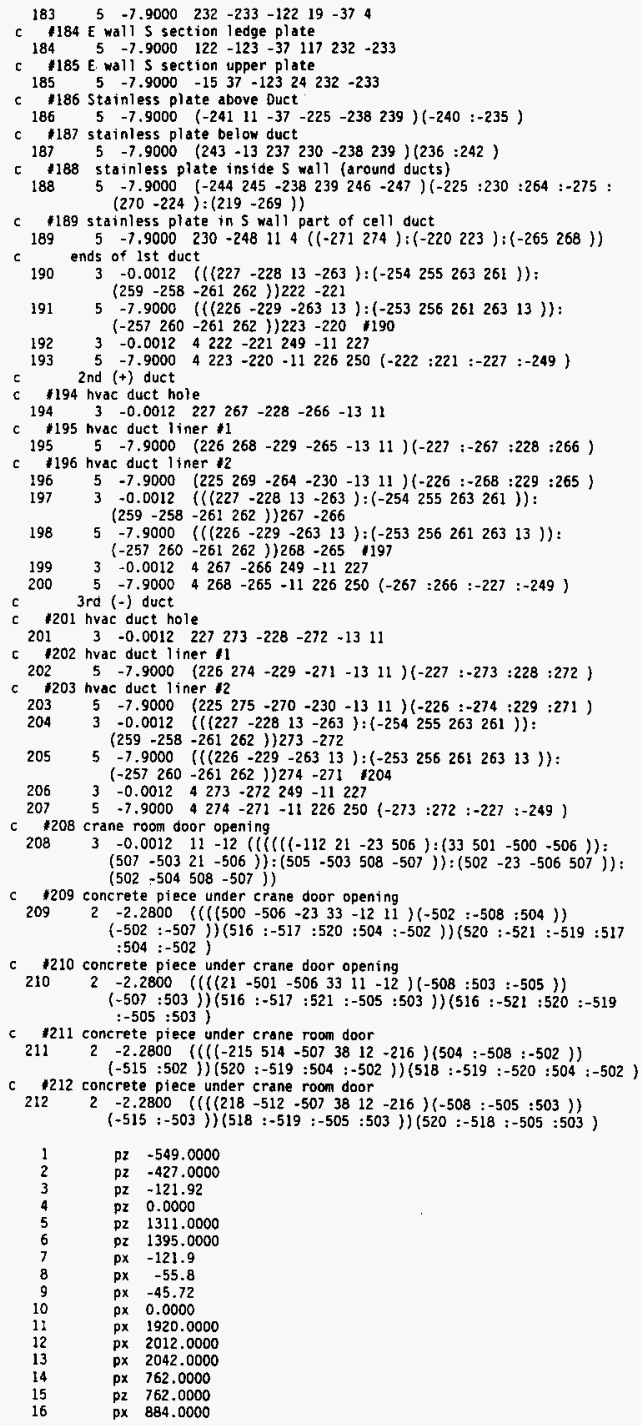


WHC-SD-WV-CN-001, Rev.0

Attachment B

Page 41 of 69

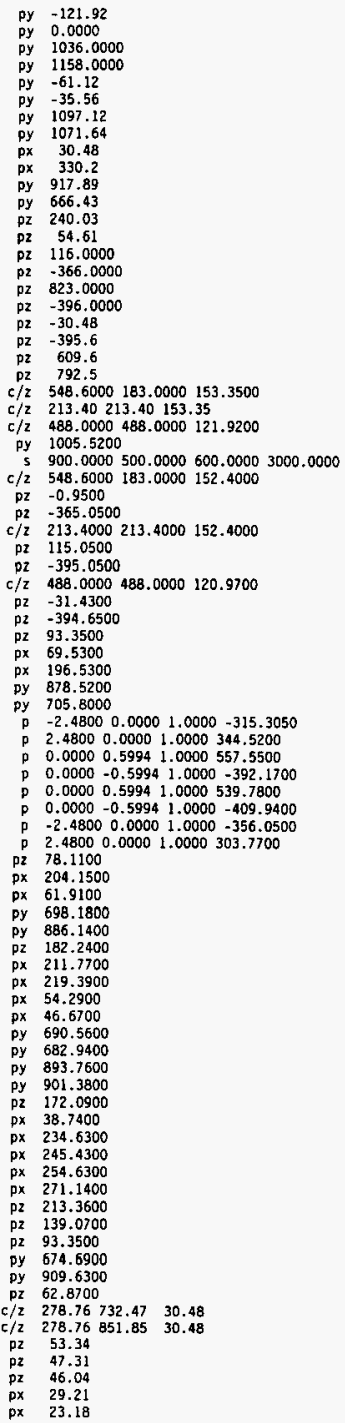


WHC-SD-WV-CN-001, Rev.0 Attachment B Page 42 of 69

\begin{tabular}{|c|c|c|c|}
\hline 99 & px & 21.91 & \\
\hline 100 & $p x$ & 331.47 & \\
\hline 101 & px & 337.50 & \\
\hline 102 & px & 338.77 & \\
\hline 103 & py & 665.16 & \\
\hline 104 & py & 659.13 & \\
\hline 105 & py & 657.86 & \\
\hline 106 & py & $\begin{array}{l}919.16 \\
925.19\end{array}$ & \\
\hline $\begin{array}{l}107 \\
108\end{array}$ & py & $\begin{array}{l}925.19 \\
926.46\end{array}$ & \\
\hline $\begin{array}{l}108 \\
109\end{array}$ & $\begin{array}{l}\text { py } \\
\text { py }\end{array}$ & $\begin{array}{l}926.46 \\
-127.16\end{array}$ & \\
\hline 110 & py & 1163.16 & \\
\hline 111 & p2 & 457.20 & \\
\hline 112 & pz & 1219.22 & \\
\hline 113 & py & 594.04 & \\
\hline 114 & py & 990.28 & \\
\hline 115 & py & 30.48 & \\
\hline $\begin{array}{l}116 \\
117\end{array}$ & px & -0.95 & \\
\hline $\begin{array}{l}117 \\
118\end{array}$ & p2 & 608.65 & \\
\hline $\begin{array}{l}118 \\
119\end{array}$ & $p x$ & -46.67 & \\
\hline $\begin{array}{l}119 \\
120\end{array}$ & py & -0.95 & \\
\hline $\begin{array}{l}120 \\
121\end{array}$ & $\begin{array}{l}p y \\
p x\end{array}$ & $\begin{array}{l}-36.51 \\
685.80\end{array}$ & \\
\hline 122 & py & 1036.95 & \\
\hline 123 & py & 1072.59 & \\
\hline 124 & $p x$ & 822.96 & \\
\hline 125 & $p z$ & 252.73 & \\
\hline 126 & $p z$ & 284.61 & \\
\hline 127 & $p z$ & 302.33 & \\
\hline 128 & $p z$ & 304.48 & \\
\hline 129 & py & 568.64 & \\
\hline 130 & py & 1015.68 & \\
\hline 131 & py & 1118.08 & \\
\hline 132 & py & $\begin{array}{l}-82.08 \\
487.68\end{array}$ & \\
\hline 133 & $p z$ & $\begin{array}{l}487.68 \\
1238.29\end{array}$ & \\
\hline $\begin{array}{l}134 \\
135\end{array}$ & $\begin{array}{l}p 2 \\
p x\end{array}$ & & \\
\hline $\begin{array}{l}135 \\
136\end{array}$ & $\begin{array}{l}p x \\
p x\end{array}$ & $\begin{array}{l}2057.24 \\
2090.26\end{array}$ & \\
\hline 137 & $\begin{array}{l}p x \\
p x\end{array}$ & $\begin{array}{l}2090.20 \\
2027.24\end{array}$ & \\
\hline 138 & $p x$ & 2050.10 & \\
\hline 139 & pz & -327.66 & \\
\hline 140 & pz & -28.57 & \\
\hline 141 & $e / z$ & $278.13 \quad 851.88$ & 30.48 \\
\hline 142 & $c / 2$ & 278.13732 .44 & 30.48 \\
\hline 143 & $t / 2$ & 397.57732 .44 & 30.48 \\
\hline 144 & $c / 2$ & 397.57851 .88 & 30.48 \\
\hline 145 & $c / 2$ & 278.13851 .88 & 30.14 \\
\hline 146 & $c / 2$ & 278.13732 .44 & 30.14 \\
\hline 147 & $6 / 2$ & 397.57851 .88 & 30.14 \\
\hline 148 & $c / 2$ & 397.57732 .44 & 30.14 \\
\hline 149 & p2 & -327.18 & \\
\hline 150 & $\mathrm{pz}$ & -28.89 & \\
\hline 151 & $p z$ & -365.76 & \\
\hline 152 & $p z$ & -66.67 & \\
\hline 153 & $c / 2$ & $612.46 \quad 892.17$ & 30.48 \\
\hline 154 & $c / 2$ & 612.46825 .50 & 30.48 \\
\hline 155 & $c / 2$ & 612.46758 .82 & 30.48 \\
\hline 156 & $c / 2$ & $612.46 \quad 692.15$ & 30.48 \\
\hline 157 & $c / z$ & $684.21 \quad 892.17$ & 30.48 \\
\hline $15 B$ & $c / 2$ & $684.21 \quad 825.50$ & 30.48 \\
\hline 159 & $c / 2$ & $684.21 \quad 758.82$ & 30.48 \\
\hline 160 & $c / 2$ & 684.21692 .15 & 30.48 \\
\hline 161 & $c / z$ & $612.46 \quad 892.17$ & 30.14 \\
\hline 162 & $c / 2$ & 612.46825 .50 & 30.14 \\
\hline 163 & $c / 2$ & 612.46758 .82 & 30.14 \\
\hline 164 & $c / z$ & $612.46 \quad 692.15$ & 30.14 \\
\hline 165 & $c / 2$ & 684.21892 .17 & 30.14 \\
\hline 166 & $c / z$ & 684.21825 .50 & 30.14 \\
\hline 167 & $c / 2$ & $684.21 \quad 758.82$ & 30.14 \\
\hline 168 & $c / z$ & $684.21 \quad 692.15$ & 30.14 \\
\hline 169 & pz & -66.99 & \\
\hline 170 & $p z$ & -365.28 & \\
\hline 171 & $p x$ & 500.0000 & \\
\hline 172 & $c / z$ & 994.17940 .75 & 30.48 \\
\hline 173 & $c / z$ & 1066.56940 .75 & 30.48 \\
\hline 174 & $c / z$ & 1569.48959 .80 & 30.48 \\
\hline 176 & $c / 2$ & 1272.30837 .88 & 30.48 \\
\hline 177 & $c / z$ & 1363.74837 .88 & 30.48 \\
\hline 178 & $c / z$ & 1363.74746 .44 & 30,48 \\
\hline 179 & $c / 2$ & 1272.30746 .44 & 30.48 \\
\hline 180 & $\mathbf{p z}$ & 30.48 & \\
\hline 181 & pz & 329.57 & \\
\hline
\end{tabular}




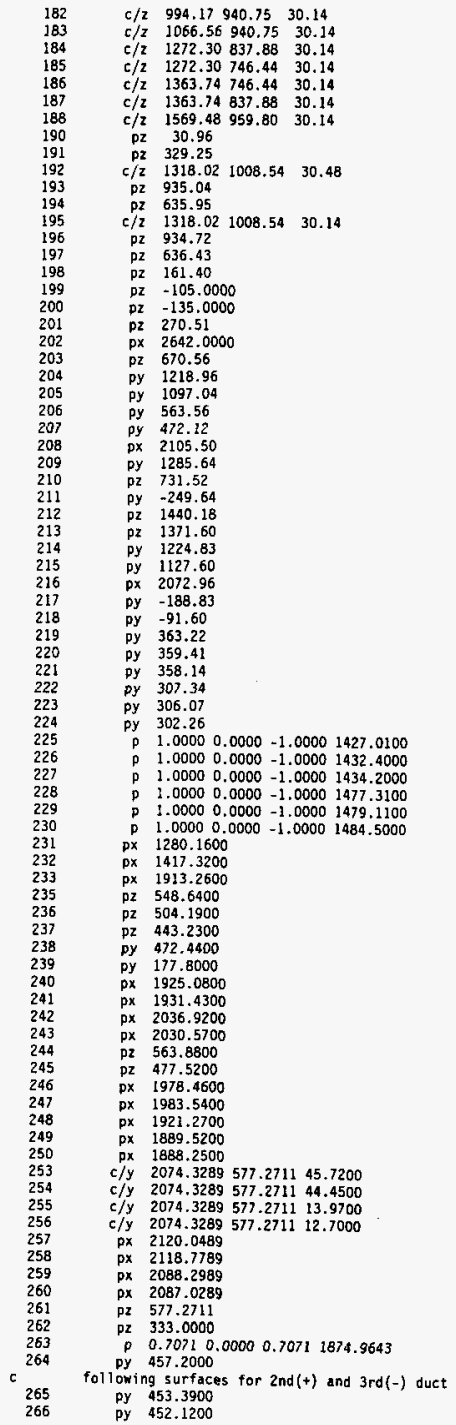




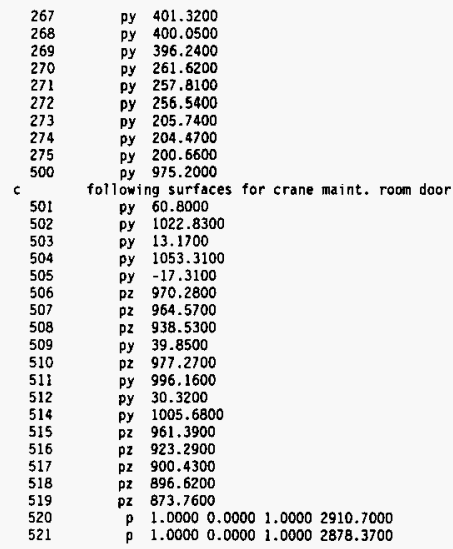

mode $p$

c i

materials

ml all hwvp dirt density $1.5 \mathrm{~g} / \mathrm{cc}$

$8000.01 p-.511 \quad 14000.01 p-.2782 \quad 20000.01 p-.0717$

$26000.01 p-.1091 \quad 13000.01 p=.0832612000 .01 p=.03142$

$19000.01 p-.0115511000 .01 p-.0202222000 .01 p-.01655$

$25000.01 \mathrm{p}-.001781$ 15000.01p -.0024

c $\quad m 2$ ordinary $H$ cancrete density $2.28 \mathrm{~g} / \mathrm{cc}$

$\begin{array}{llllllll}\text { m2 } & 1000.01 p & 0.0642 & 8000.01 p & 0.5916 & 14000.01 p & 0.2405\end{array}$

$20000.01 p \quad 0.0738 \quad 26000.01 p \quad 0.0299$

c $\quad \mathrm{m}^{3}$ air density $.0012 \mathrm{~g} / \mathrm{cc}$

$\mathrm{m3} 7000.01 \mathrm{p}-0.765 \quad 8000.01 \mathrm{p}-0.235$

c $m 4$ water density $1 \mathrm{~g} / \mathrm{cc}$

m4 $1000.01 \mathrm{p} .6667 \quad 8000.01 \mathrm{p} .3333$

c m5 $304 \mathrm{sS}$ (stainless steel) density $7.9 \mathrm{~g} / \mathrm{cc}$

m5 25000.01p $=.02 \quad 14000.01 p-.01 \quad 24000.01 \mathrm{p}-.19$

$28000.01 p-.10 \quad 26000.01 p-.68$

c m6 K-3 block density $3.83 \mathrm{~g} / \mathrm{cc}$

m6 $13000.01 \mathrm{p}-.2857 \quad 24000.01 \mathrm{p}=.2546$

$26000.01 p-.0615 \quad 8000.01 p-.3981$

c m $7 \mathrm{H}$ block melter density $3.139 \mathrm{~g} / \mathrm{cc}$

m) $\quad 13000.01 \mathrm{p}-.5096 \quad 11000.01 \mathrm{p}-.0274$

$8000.01 \mathrm{p}-.4629$

c $m 8 Z$ ( $Z$ i rmul) melter block density $2.95 \mathrm{~g} / \mathrm{cc}$

m8 $13000.01 \mathrm{p}-.369740000 .01 \mathrm{p}-.1767$

$14000.01 \mathrm{p}-.0293 \quad 8000.010-.4243$

c $m 9$ AL-66 melter ref ractory density $2.72 \mathrm{~g} / \mathrm{cc}$

m9 $13000.01 p-.5063 \quad 20000.01 p-.0157$ 26000.01p - .0109 $14000.01 p-.0027 \quad 8000.01 p-.4644$

c $\quad 10$ is melter FEED material density $1.3 \mathrm{~g} / \mathrm{cc}$

m10 26000.01p -.0345 7000.01p -.0250 11000.01p -.0604 $90000.01 p-.0136 \quad 6000.01 p=.0386 \quad 1000.01 p=.0767$ $5000.01 p-.0368 \quad 15000.01 p=.0047 \quad 14000.01 p=.0851$ $8000.010-.9255$

c mll is glass waste material density $2.7 \mathrm{~g} / \mathrm{cc}$

m11 $14000.01 \mathrm{p}-.710090000 .01 \mathrm{p}-.1100 \quad 26000.01 \mathrm{p}-.2900$ $11000.010-.2700 \quad 5000.01 p-.1100 \quad 8000.01 p-1.260$ $\mathrm{m} 12$ is iron for the doors density $7.8 \mathrm{~g} / \mathrm{cc}$

m12 26000.01p - 1.00

c m13 AL-57 melter refractory density $1.04 \mathrm{~g} / \mathrm{cc}$

m13 $13000.01 \mathrm{p}=.5075 \quad 20000.010-.0158426000 .010=.01109$ $14000.01 \mathrm{p}-.00139 \quad 8000.01 \mathrm{p}-.4642$

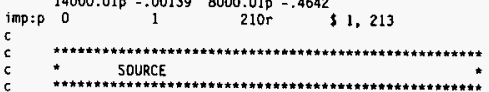


source description

ce! di pos fcel d2 axs $0,0,1$.

ext fcel d3 wht $3.91+16$

rad fcel d4 erg fcel d5

scl source cell probability distribution

sil 11830417879

8081919293

$\begin{array}{lllll}94 & 95 & 96 & 97 & 98\end{array}$

$\begin{array}{llll}126 & 127 & 128 & 129\end{array}$

$\begin{array}{llll}130 & 131 & 132 & 156\end{array}$

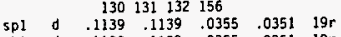

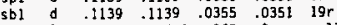

ds? $i \quad 213.4213 .4-265.5$ xyz cell centers

$548.6 \quad 183.0 \quad-235$.

133.03792 .16127 .375

$278.13851 .88=178$.

$397.57851 .88-178$

$278.13732 .44-178$.

$397.57732 .44-178$.

$612.46 \quad 692.15-216$.

$612.46758 .82-216$.

$612.46825 .5-216$.

$612.46892 .17 \cdot 216$

$684.21892 .17-216$.

$684.21825 .5-216$.

$684.21758 .82-216$.

$684.21 \quad 692.15=216$.

1056.56940 .75180 .1

994.17940 .75180 .1

$1272.3837 .88 \quad 180.1$

1272.3746 .44180 .1

1363.74746 .44180 .

1363.74837 .88180$.

$1569.48 \quad 959.8 \quad 180.1$

1318.01008 .54785 .6

ds 3 s $667819 \mathrm{r}$ S distrib. Is for ext

$516 \quad 130$.

$\begin{array}{ll}517 & 93.35 \\ \text { si } 8 & 149 .\end{array}$

ds 4 s 991011 19r\$distrib. Is for radius

$519 \quad 152.4$

5110105

si11 30.14

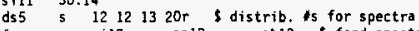

si12 spl2 sb12 s feed spectrum

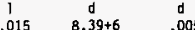

$\begin{array}{lll}.025 & 3.15+6 & .002\end{array}$

$\begin{array}{lll}.035 & 1.56+7 & .01\end{array}$

$\begin{array}{lll}.045 \quad 1.83+6 & .001\end{array}$

$.055-1.51+6$

.065

$\begin{array}{lll}.075 & 6.94+5 & .001\end{array}$

$.085 \quad 7.22+5 \quad .001$

$\begin{array}{lll}.095 & 5.51+5 & .001\end{array}$

$.15-3.32+6 \quad .004$

$\begin{array}{lll}.25 & 9.45+5 & .001\end{array}$

$.35 \quad 4.05+5 \quad .001$

$\begin{array}{lll}.475 & 3.76+5 & .001 \\ .65 & 1.71+8 & 1.0\end{array}$

$\begin{array}{lll}.825 & 3.80+5 & .01\end{array}$

$1.0 \quad 5.66+5 \quad .05$

$\begin{array}{lll}1.0 & 5.66+5 & .05 \\ 1.225 & 7.51+5 & .1\end{array}$

$\begin{array}{lll}1.475 & 6.27+4 & .05\end{array}$

$\begin{array}{lll}1.7 & 6.27+4 & .05\end{array}$

1.9 $\quad 1.09+4 \quad 1$

2.3

$2.14-4 \quad .001$

$3.27-4 \quad .002$

$\begin{array}{ll}9.25+1 & .05 \\ 2.90-3 & .001\end{array}$

.90-3 .001

d d

$2.83+7$

$1.21+7 \quad .003$

$4.02+7 \quad .01$

$6.18+6 \quad .002$

$4.94+6 \quad .002$

$2.94+6 \quad .002$

$\begin{array}{ll}2.64+6 & .002 \\ 2.61+6 & .002\end{array}$

q ghass spectrum 
WHC-SD-WV-CN-001, Rev.0 Attachment B

Page 46 of 69

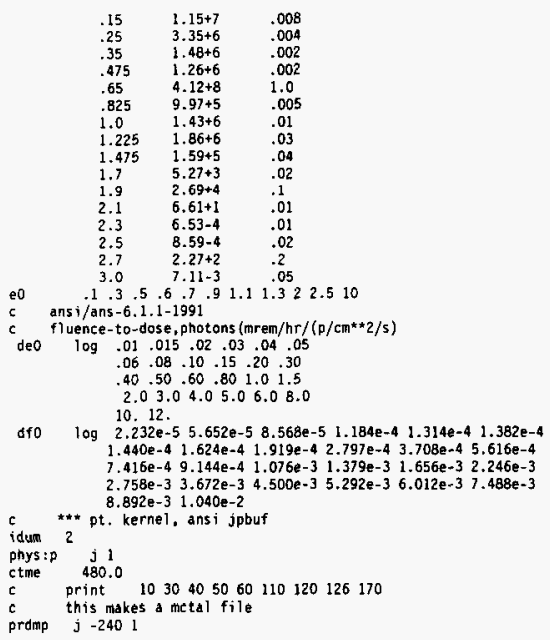




\section{Input file for the canister configuration without the cart or crane sources} (15 canisters):

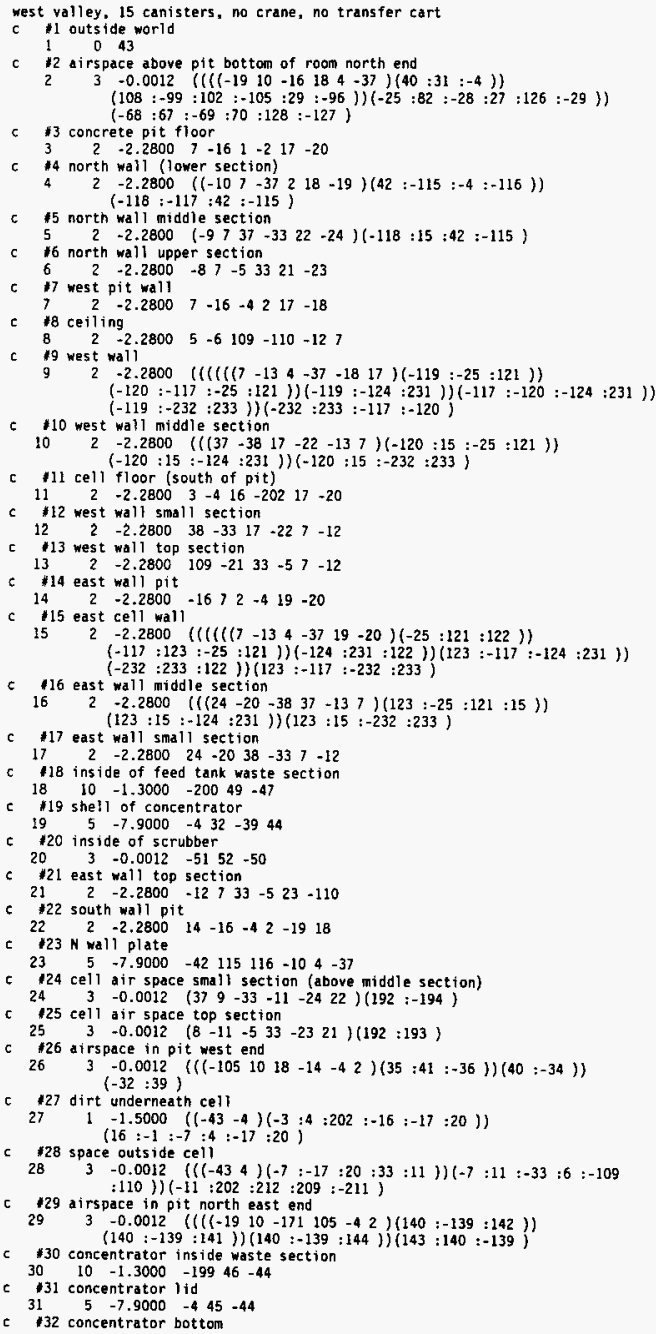




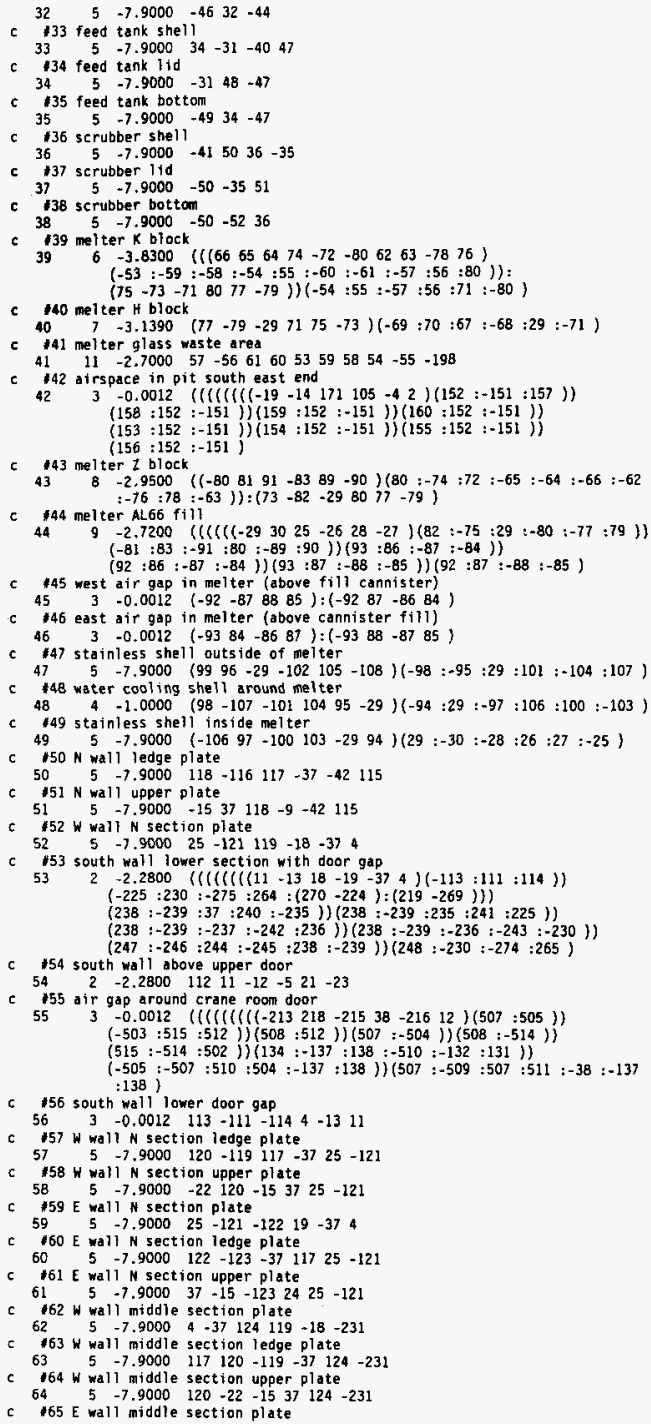




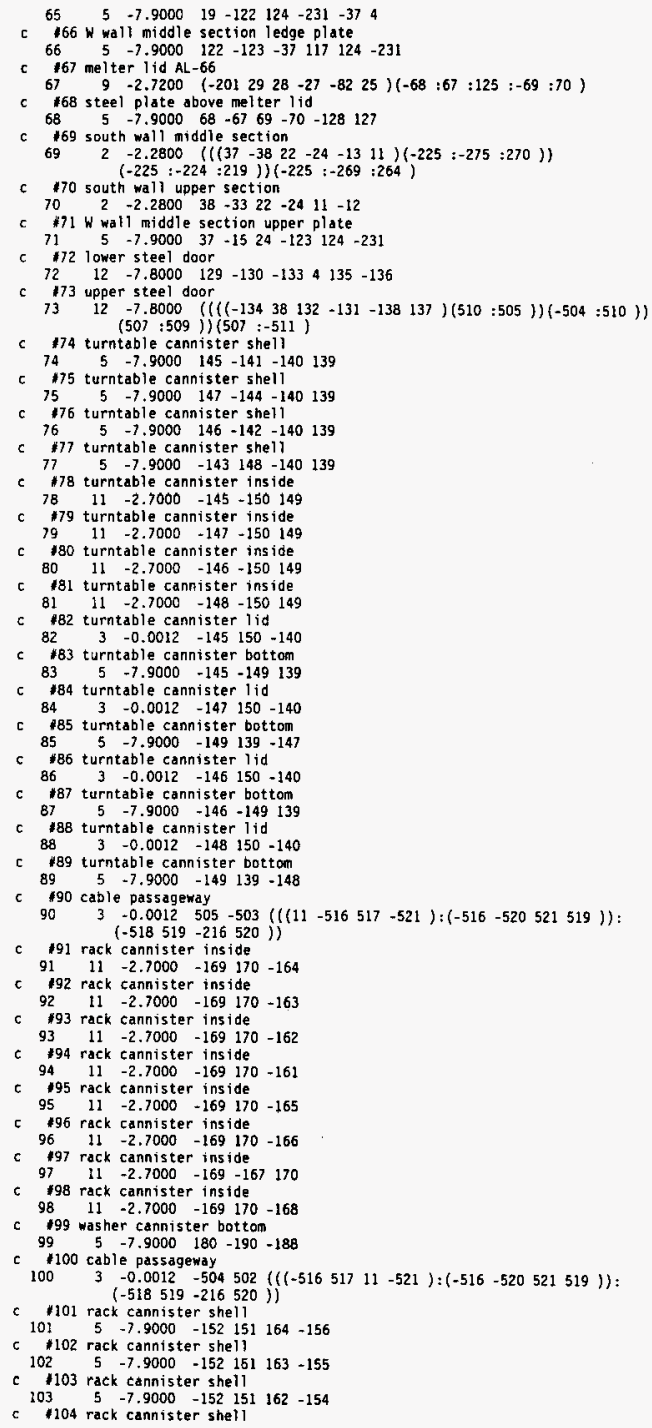




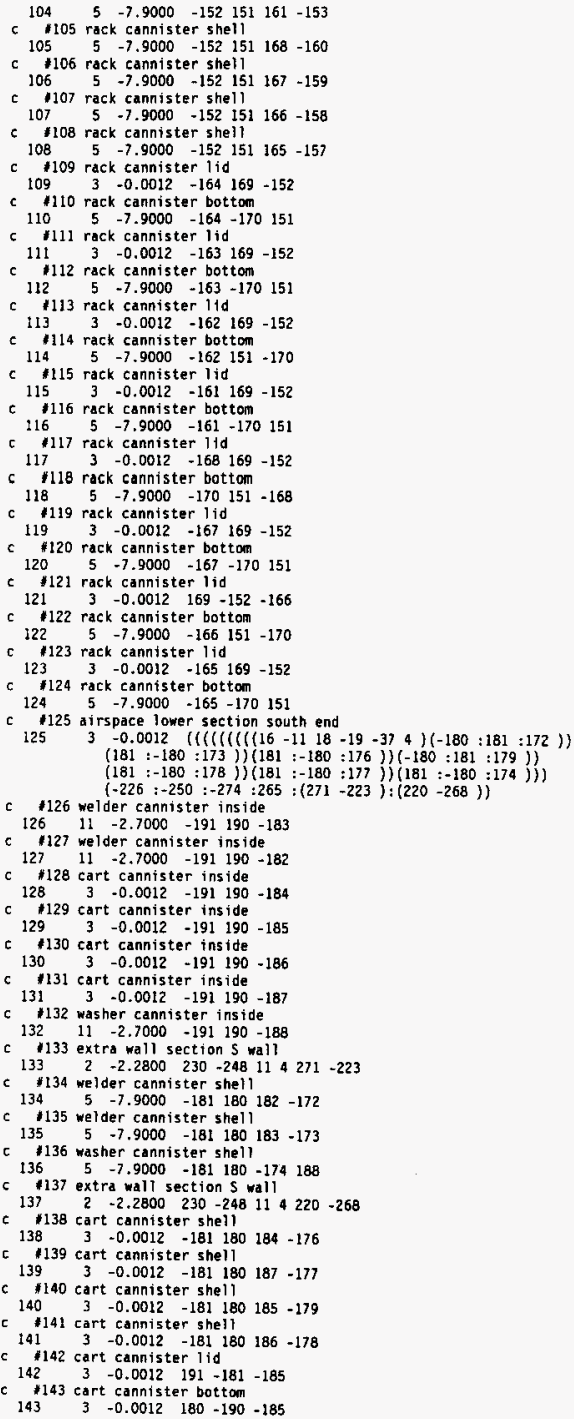


c 144 cart cannister 11 id

$144 \quad 3-0.0012-181 \quad 191-186$

c 145 cart cannister battom

$1453-0.0012-190 \quad 180-186$

c 146 cart cannister lid

$\begin{array}{lllllll}146 & 3 & -0.0012 & 19] & -181 & -184\end{array}$

c 147 cart cannister botton

$1473-0.0012 \quad 180-190-184$

c 148 cart cannister 1 id

$\begin{array}{llllll}148 & 3 & -0.0012 & 191 & -181 & -187\end{array}$

c 149 cart cannister bottom

$1993 \begin{array}{lllll}149 & -0.0012 & 180 & -190 & -187\end{array}$

c 150 welder cannister lid

$\begin{array}{llllll}150 \quad 3 & -0.0012 \quad 191-181 & -182\end{array}$

c 151 welder cannister bottom

$15155-7.9000 \quad 180-190-182$

c 152 velder cannister lid

$1523-0.0012191-181-183$

c $\$ 153$ welder cannister botton

$153 \quad 5-7.9000 \quad 180-190-183$

- 154 washer cannister lid

$\begin{array}{lllllll}154 & 5 & -7,9000 & 191 & -181 & -188\end{array}$

c 155 crane hook cannister shell

$1553 \quad-0.0012 \quad-193-192 \quad 195194$

c \$156 crane hook cannister inside

$\begin{array}{llllll}156 & 3 & -0.0012 & 197 & -195 & -195\end{array}$

c $\$ 157$ crane hook cannister lid

$15730.0012 \quad 196-193-195$

c 158 crane hook cannister botton

$\begin{array}{llllll}158 & 3 & -0.0012 & -197 & 194 & -195\end{array}$

c 159 melter airspace inside

$\begin{array}{lllllllllll}159 & 3 & -0.0012 & -125 & 68 & -57 & 71 & 69 & -70\end{array}$

c 1160 melter airspace inside

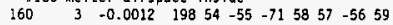

c 161 concentrator air space

$\begin{array}{llllll}161 & 3 & -0.0012 & -45 & -44 & 199\end{array}$

c 162 feed tank air space

$\begin{array}{lllll}162 & 3 & -0.0012 & -48 & -47 \\ 200\end{array}$

c 163 melter lid AL -57

$\begin{array}{lllllllll}163 & 13 & -1.0400 & 201 & -126 & 28 & -27 & -82 & 25\end{array}$

c 164 Transfer tunnel $W$ wall

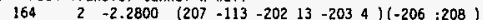

\& 165 crane maintenance room floor

$1652 \quad-2.2800(-209-202 \quad 211 \quad 11-210203)(20:-17: 13)$

c 166 transfer tunnel $E$ wall

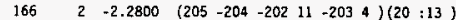

c 1167 crane room ceiling

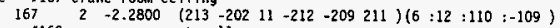

c $\$ 168$ crane room east wall

$\begin{array}{lllllllll}168 & 2 & -2.2800 & -202 & 11 & -209 & 214 & -213 & 210\end{array}$

c 169 crane room W wall

$169 \quad 2-2.2800 \quad 211-217-20211210-213$

c $\$ 170$ ledge underneath crane room door

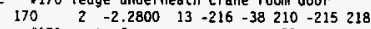

c 111 part of crane room east wall

$1712 \quad 2-2.2800(((-216 \quad 11-214 \quad 215-213 \quad 210)(110: 12:-33))$

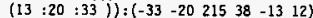

$\$ 172$ part of crane roon vest wall

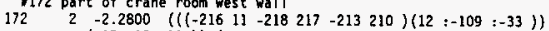

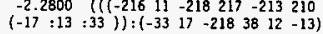

- $\$ 173$ crane room a ir spoce

$\begin{array}{llllllllll}173 & 3 & -0.0012 & -214 & 217 & -202 & 216 & -213 & 210\end{array}$

c $\$ 174$ airspace $S$ of cell $W$ of transfer tunnle, beyond 191

$1743-0.0012(-20211211-207-2034)(13:-17)((((-226: 229: 263)$ $(253:-256:-261:-263))(257:-260: 261:-262)):-274: 265$ : $(271-223):(220-268))$

c $\$ 175$ airspace $E$ of transfer tunnel

$175 \quad 3=0.0012-209204-20211-2034$

c 176 transfer tunnel airspace

$176 \quad 3-0.0012((20613-205-202-2034)(-208: 113))$ $(-129:-135 \cdot 136 \cdot 130: 133)$

c $\$ 177$ hvac duct hole

$\begin{array}{lllllllll}177 & 3 & -0.0012 & 227 & 222 & -228 & -221 & -13 & 11\end{array}$

c 178 huac duet liner 1

$17855-7.9000 \quad\left\{\begin{array}{llllll}226 & 223 & -229 & -220-13 & 11)(-227:-222: 228: 221\}\end{array}\right.$

c $\$ 179$ hrac duct liner 12

$1795 \quad-7.9000(225224-219-230-1311)(-226:-223: 229: 220)$

c $180 \mathrm{~W}$ wall S section plate

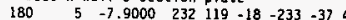

c $181 \mathrm{~W}$ wall $\mathrm{s}$ section ledge plate

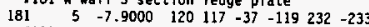

c $182 \mathrm{Wwal}$ s section upper plate 


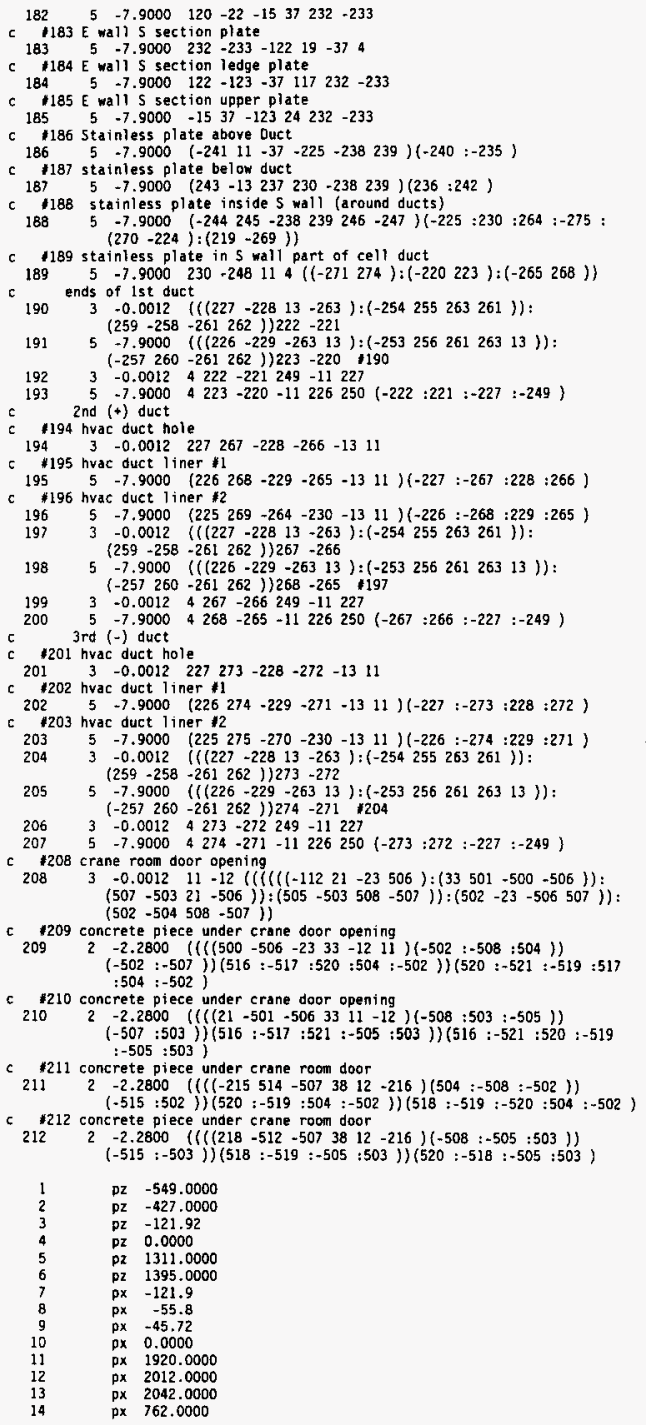


WHC-SD-WV-CN-001, Rev.0

Attachment B

Page 53 of 69

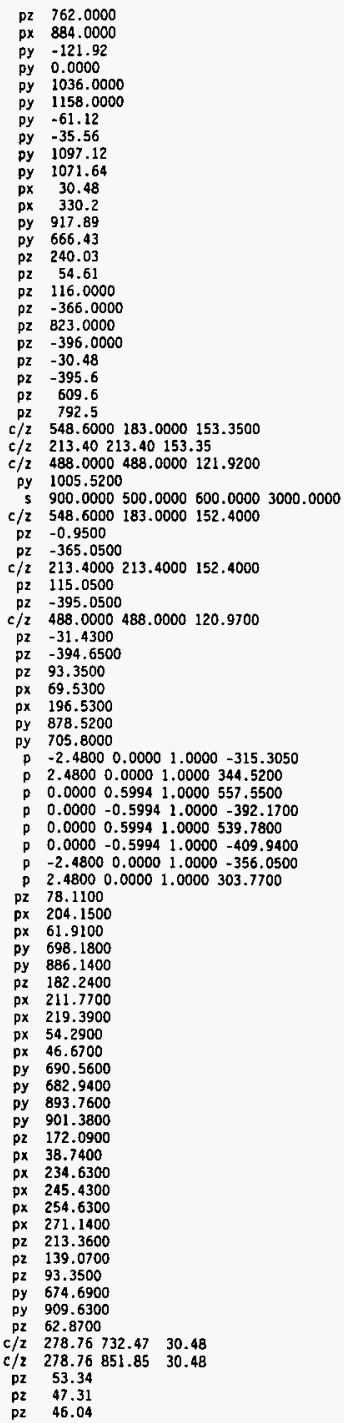


WHC-SD-WV-CN-001, Rev.0

Attachment B

Page 54 of 69

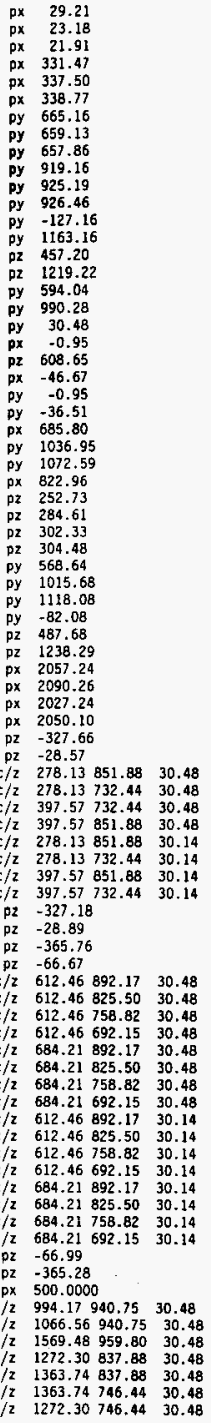




\section{WHC-SD-WV-CN-001, Rev.0 \\ Attachment B \\ Page 55 of 69}

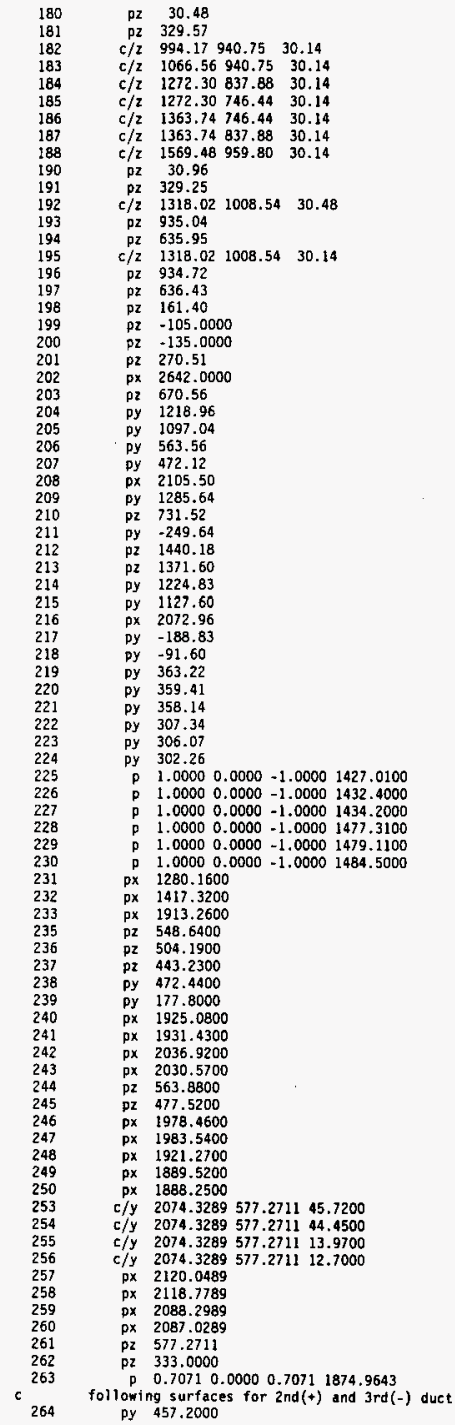




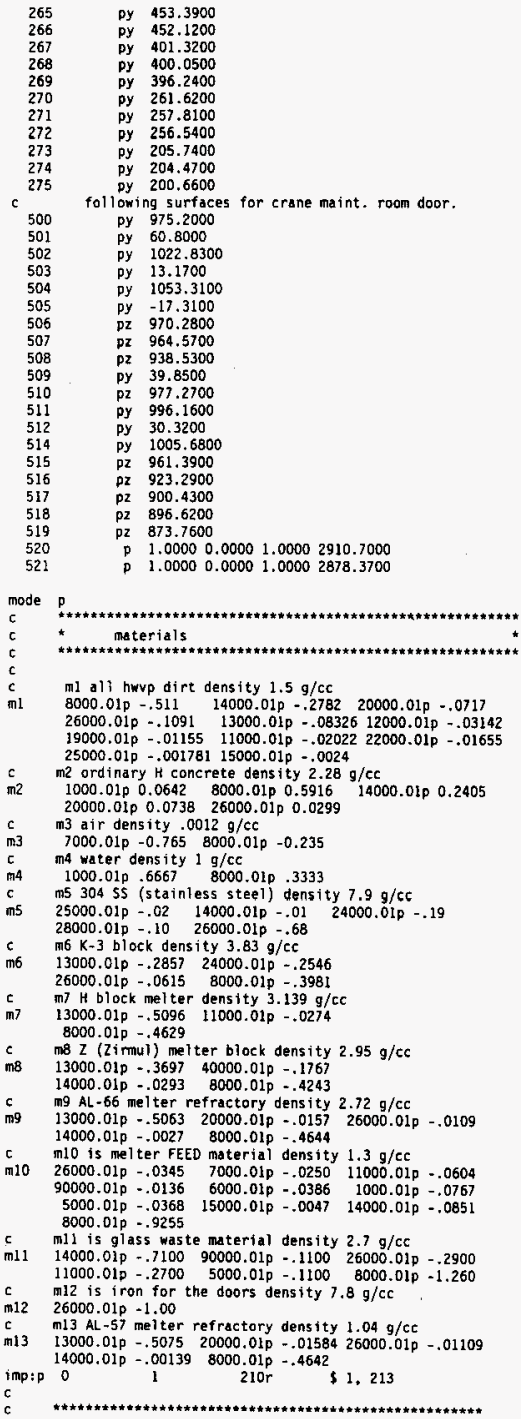




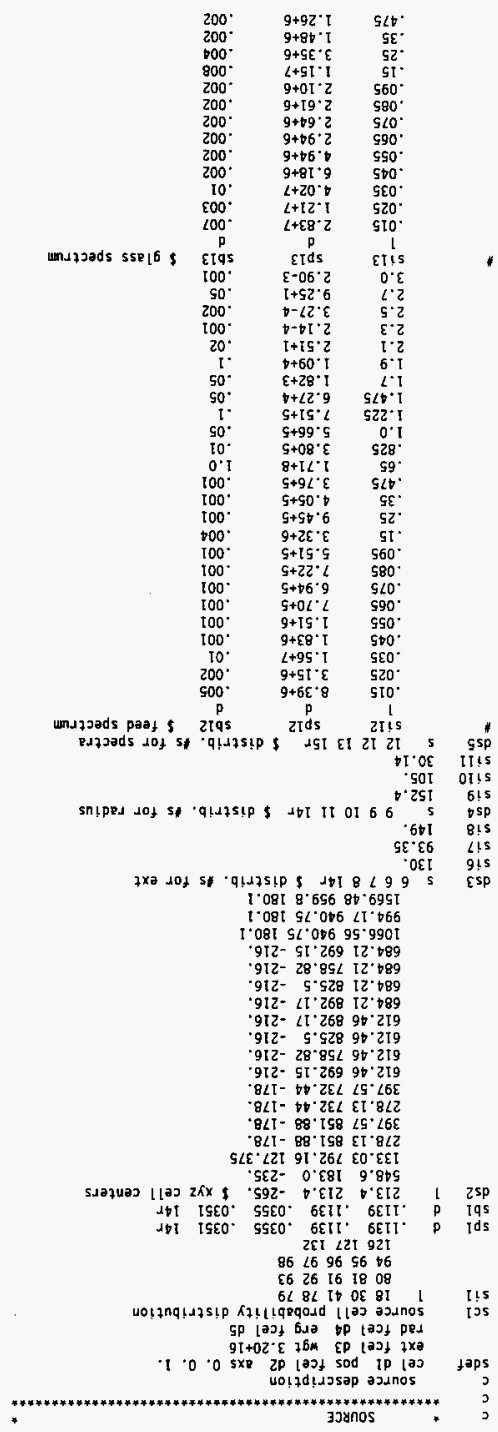

69 to $\angle S$ 26ed

g 7иวшцวе77甘

0 '^əУ '[00-N]- $M M-O S-J H M$ 
WHC-SD-WV-CN-001, Rev.0

Attachment B

Page 58 of 69

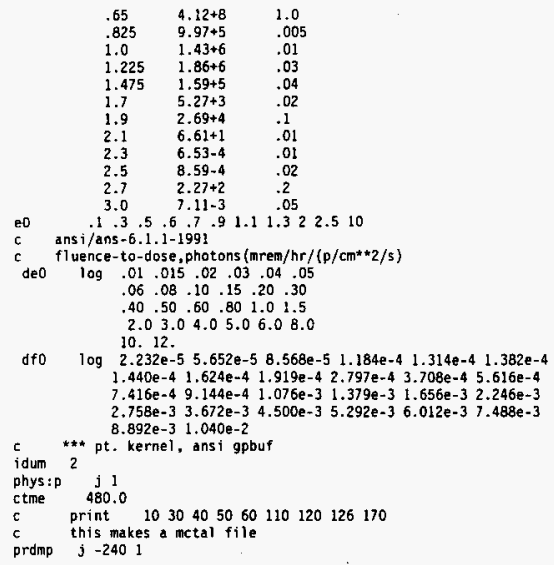


The detector cards for the six input files were not included in this listing due to their size. One of the detector cards from one of the files is included below as an example:

\author{
fc395 Dose point at 2121 (PV-Wave coordinates) \\ $f 395: p \quad-1123.0000002121 .00000091 .4400020$ \\ $-1038.0000002121 .00000091 .4400020$ \\ $-953.0000002121 .00000091 .4400020$ \\ $-868.0000002121 .00000091 .4400020$ \\ $-783.0000002121 .00000091 .4400020$ \\ $-698.0000002121 .00000091 .4400020$ \\ $-613.0000002121 .00000091 .4400020$ \\ $-528.0000002121 .00000091 .4400020$ \\ $-443.0000002121 .00000091 .4400020$ \\ $-358.0000002121 .00000091 .4400020$ \\ $-273.0000002121 .00000091 .4400020$

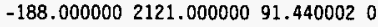 \\ $-122.0000002121 .00000091 .4400020$ \\ 1.0000002121 .00000091 .4400020 \\ 67.0000002121 .00000091 .4400020 \\ 152.0000002121 .00000091 .4400020 \\ 237.0000002121 .00000091 .4400020 \\ 322.0000002121 .00000091 .4400020 \\ 407.0000002121 .00000091 .4400020 \\ 492.0000002121 .00000091 .4400020 \\ 577.0000002121 .00000091 .4400020 \\ 662.0000002121 .00000091 .4400020 \\ 747.0000002121 .00000091 .4400020 \\ 832.0000002121 .00000091 .4400020 \\ 917.0000002121 .00000091 .4400020 \\ 1002.0000002121 .00000091 .4400020 \\ 1087.0000002121 .00000091 .4400020 \\ 1172.0000002121 .00000091 .4400020 \\ 1257.0000002121 .00000091 .4400020 \\ 1342.0000002121 .00000091 .4400020 \\ 1427.0000002121 .00000091 .4400020 \\ 1512.0000002121 .00000091 .4400020 \\ 1597.0000002121 .00000091 .4400020 \\ 1682.0000002121 .00000091 .4400020 \\ 1767.0000002121 .00000091 .4400020 \\ 1852.0000002121 .00000091 .4400020 \\ 1919.0000002121 .00000091 .4400020 \\ 2043.0000002121 .00000091 .4400020 \\ 2107.0000002121 .00000091 .4400020 \\ 2192.0000002121 .00000091 .4400020 \\ 2277.0000002121 .00000091 .4400020 \\ 2362.0000002121 .00000091 .4400020 \\ 2447.0000002121 .00000091 .4400020 \\ 2532.0000002121 .00000091 .4400020 \\ 2617.0000002121 .00000091 .4400020 \\ 2702.0000002121 .00000091 .4400020 \\ 2787.0000002121 .00000091 .4400020 \\ 2872.0000002121 .00000091 .4400020 \\ 2957.0000002121 .00000091 .4400020 \\ 3042.0000002121 .00000091 .4400020
}


One detector was created at each $X$ and $Y$ location along a grid. Each level had a different grid. These grids are described in Table B-1.

Table B-1.

\begin{tabular}{|c|c|c|c|c|c|c|c|}
\hline \multicolumn{2}{|c|}{ 103' level: } & \multicolumn{2}{|c|}{$113.25^{\prime}$ level: } & \multicolumn{2}{|c|}{127 ' level: } & \multicolumn{2}{|c|}{$134.5^{\prime}$ level: } \\
\hline \multicolumn{2}{|c|}{$Z=91.44$} & \multicolumn{2}{|c|}{$Z=403.86$} & \multicolumn{2}{|c|}{$z=822.96$} & \multicolumn{2}{|c|}{$z=1051.56$} \\
\hline$Y=$ & $x=$ & $\gamma=$ & $x=$ & $\gamma=$ & $x=$ & $\gamma=$ & $x=$ \\
\hline-1155 & -1123 & -1155 & -1123 & -1155 & -1123 & -1155 & -1123 \\
\hline-1071 & -1038 & -1071 & -1038 & -1071 & -1038 & -1071 & -1038 \\
\hline-987 & -953 & -987 & -953 & -987 & -953 & -987 & -953 \\
\hline-903 & -868 & -903 & -868 & -903 & -868 & -903 & -868 \\
\hline-819 & -783 & -819 & -783 & -819 & -783 & -819 & -783 \\
\hline-735 & -698 & -735 & -698 & -735 & -698 & -735 & -698 \\
\hline-651 & -613 & -651 & -613 & -651 & -613 & -651 & -613 \\
\hline-567 & -528 & -567 & -528 & -567 & -528 & -567 & -528 \\
\hline-483 & -443 & -483 & -443 & -483 & -443 & -483 & -443 \\
\hline-399 & -358 & -399 & -358 & -399 & -358 & -399 & -358 \\
\hline-315 & -273 & -315 & -273 & -315 & -273 & -315 & -273 \\
\hline-231 & -188 & -231 & -188 & -250 & -188 & -250 & -188 \\
\hline-147 & -122 & -147 & -122 & -188 & -122 & -188 & -122 \\
\hline-122 & 1 & -122 & 1 & -128 & -45 & -128 & -45 \\
\hline 1 & 67 & 1 & 67 & -30 & 67 & -30 & 67 \\
\hline 105 & 152 & 105 & 152 & 105 & 152 & 105 & 152 \\
\hline 189 & 237 & 189 & 237 & 189 & 237 & 189 & 237 \\
\hline 273 & 322 & 273 & 322 & 273 & 322 & 273 & 322 \\
\hline 357 & 407 & 357 & 407 & 357 & 407 & 357 & 407 \\
\hline 441 & 492 & 441 & 492 & 441 & 492 & 441 & 492 \\
\hline 525 & 577 & 525 & 577 & 525 & 577 & 525 & 577 \\
\hline 609 & 662 & 609 & 662 & 609 & 662 & 609 & 662 \\
\hline 693 & 747 & 693 & 747 & 693 & 747 & 693 & 747 \\
\hline 777 & 832 & 777 & 832 & 777 & 832 & 777 & 832 \\
\hline 861 & 917 & 861 & 917 & 861 & 917 & 861 & 917 \\
\hline 945 & 1002 & 945 & 1002 & 945 & 1002 & 945 & 1002 \\
\hline 1035 & 1087 & 1035 & 1087 & 1066 & 1087 & 1066 & 1087 \\
\hline 1159 & 1172 & 1159 & 1172 & 1164. & 1172 & 1164 & 1172 \\
\hline 1219 & 1257 & 1219 & 1257 & $1224^{\circ}$ & 1257 & 1224 & 1257 \\
\hline 1281 & 1342 & 1281 & 1342 & 1286 & 1342 & 1286 & 1342 \\
\hline 1365 & 1427 & 1365 & 1427 & 1365 & 1427 & 1365 & 1427 \\
\hline 1449 & 1512 & 1449 & 1512 & 1449 & 1512 & 1449 & 1512 \\
\hline 1533 & 1597 & 1533 & 1597 & 1533 & 1597 & 1533 & 1597 \\
\hline 1617 & 1682 & 1617 & 1682 & 1617 & 1682 & 1617 & 1682 \\
\hline 1701 & 1767 & 1701 & 1767 & 1701 & 1767 & 1701 & 1767 \\
\hline 1785 & 1852 & 1785 & 1852 & 1785 & 1919 & 1785 & 1919 \\
\hline 1869 & 1919 & 1869 & 1919 & 1869 & 2027 & 1869 & 2027 \\
\hline 1953 & 2043 & 1953 & 2043 & 1953 & 2051 & 1953 & 2051 \\
\hline 2037 & 2107 & 2037 & 2107 & 2037 & 2073 & 2037 & 2073 \\
\hline 2121 & 2192 & 2121 & 2192 & 2121 & 2192 & 2121 & 2192 \\
\hline & 2277 & & 2277 & & 2277 & & 2277 \\
\hline & 2362 & & 2362 & & 2362 & & 2362 \\
\hline & 2447 & & 2447 & & 2447 & & 2447 \\
\hline & 2532 & & 2532 & & 2532 & & 2532 \\
\hline & 2617 & & 2617 & & 2617 & & 2617 \\
\hline & 2702 & & 2702 & & 2702 & & 2702 \\
\hline & 2787 & & 2787 & & 2787 & & 2787 \\
\hline & 2872 & & 2872 & & 2872 & & 2872 \\
\hline & 2957 & & 2957 & & 2957 & & 2957 \\
\hline & 3042 & & 3042 & & 3042 & & 3042 \\
\hline
\end{tabular}


WHC-SD-WV-CN-001, Rev. 0

Attachment $\mathrm{C}$

Page 61 of 69

Attachment $C$.

ISOSHLD output files. 


\section{ISOSHLD 4.0}

UNIX/IBM PC (September 1994)

Radiation Physics \& Shielding Westinghouse Handford Company Richland, WA 99352

Initializing photon library: REAC*4 8/19/92 14:46:17 Initializing mass attenuation library: attnuate.lib

SHIELD COMPOSITION, $g / c c$

Shield 1 Shield 2 Shield 3 Shield 4 Shield 5

\begin{tabular}{ll}
\hline SI & $7.100 E-01$ \\
TH & $1.100 E-01$ \\
FE & $2.900 E-01$ \\
NA & $2.700 E-01$ \\
B & $1.100 E-01$ \\
O & $1.260 E+00$
\end{tabular}

> Retrieving data from ISODAT.QQQ

SRC FOR $1 \mathrm{~g}$ of WV GLASS

Feb $1,95 \quad 12: 58: 47 \quad S$

Scale factor $=1.000 E+00$

Isotopic Source Term (unscaled)

$\begin{array}{llllll}\text { FE- 55 } & 2.930 E-07 & \text { CO- 60 } & 7.250 E-07 & \text { NI- 59 } & 2.190 E-07 \\ \text { SE- 79 } & 7.290 E-09 & \text { SR- 90 } & 1.200 E-02 & \text { Y }-90 & 1.200 E-02 \\ \text { ZR-93 } & 5.630 E-07 & \text { NB- 93* } & 4.190 E-07 & \text { TC- 99 } & 2.250 E-07 \\ \text { RU-106 } & 4.750 E-10 & \text { RH-106 } & 4.760 E-10 & \text { PD-107 } & 2.280 E-08 \\ \text { CD-113* } & 3.300 E-06 & \text { SN-121* } & 3.320 E-08 & \text { SN-126 } & 2.150 E-07 \\ \text { SB-125 } & 3.350 E-06 & \text { SB-126 } & 3.010 E-08 & \text { SB-126* } & 2.150 E-07 \\ \text { TE-125* } & 8.210 E-07 & \text { CS-134 } & 1.420 E-06 & \text { CS-135 } & 3.340 E-07\end{array}$


WHC-SD-WV-CN-001, Rev.0

Attachment $\mathrm{C}$

Page 63 of 69

$\begin{array}{llllll}\text { CS-137 } & 1.300 E-02 & \text { BA-137* } & 1.230 E-02 & \text { CE-144 } & 6.440 E-12 \\ \text { PR-144 } & 6.450 E-12 & \text { PM-146 } & 1.050 E-08 & \text { PM-147 } & 3.710 E-05 \\ \text { SM-151 } & 1.670 E-04 & \text { EU-152 } & 5.560 E-07 & \text { EU-154 } & 1.220 E-04 \\ \text { EU-155 } & 2.130 E-05 & \text { TL-207 } & 1.940 E-08 & \text { TL-208 } & 6.190 E-09 \\ \text { PB-209 } & 4.440 E-10 & \text { PB-211 } & 1.950 E-08 & \text { PB-212 } & 1.720 E-08 \\ \text { BI-211 } & 1.950 E-08 & \text { BI-212 } & 1.720 E-08 & \text { BI-213 } & 4.440 E-10 \\ \text { PO-215 } & 1.950 E-08 & \text { PO-216 } & 1.720 E-08 & \text { RN-219 } & 1.950 E-08 \\ \text { RN-220 } & 1.720 E-08 & \text { FR-221 } & 4.440 E-10 & \text { FR-223 } & 2.690 E-10 \\ \text { RA-223 } & 1.950 E-08 & \text { RA-224 } & 1.720 E-08 & \text { RA-225 } & 4.440 E-10 \\ \text { RA-228 } & 3.260 E-09 & \text { AC-225 } & 4.440 E-10 & \text { AC-227 } & 1.950 E-08 \\ \text { AC-228 } & 3.260 E-09 & \text { TH-227 } & 1.920 E-08 & \text { TH-228 } & 1.720 E-08 \\ \text { TH-229 } & 4.440 E-10 & \text { TH-230 } & 1.210 E-10 & \text { TH-231 } & 1.960 E-10 \\ \text { TH-232 } & 3.390 E-09 & \text { TH-234 } & 1.650 E-09 & \text { PA-231 } & 3.140 E-08 \\ \text { PA-233 } & 4.850 E-08 & \text { PA-234* } & 1.650 E-09 & \text { U -232 } & 1.360 E-08 \\ \text { U -233 } & 1.870 E-08 & \text { U -234 } & 8.950 E-09 & \text { U -235 } & 1.960 E-10 \\ \text { U -236 } & 5.730 E-10 & \text { U -238 } & 1.650 E-09 & \text { NP-236 } & 1.960 E-08 \\ \text { NP-237 } & 4.850 E-08 & \text { NP-239 } & 7.170 E-07 & \text { PU-236 } & 1.740 E-09 \\ \text { PU-238 } & 1.640 E-05 & \text { PU-239 } & 3.360 E-06 & \text { PU-240 } & 2.470 E-06 \\ \text { PU-241 } & 1.250 E-04 & \text { PU-242 } & 3.360 E-09 & \text { AM-241 } & 1.110 E-04 \\ \text { AM-242 } & 5.940 E-07 & \text { AM-242* } & 5.970 E-07 & \text { AM-243 } & 7.170 E-07 \\ \text { CM-242 } & 4.930 E-07 & \text { CM-243 } & 2.400 E-07 & \text { CM-244 } & 1.260 E-05 \\ \text { CM-245 } & 1.820 E-09 & \text { CM-246 } & 2.090 E-10 & & \end{array}$

Case Title: Brems. generation in GLASS

\begin{tabular}{|c|c|c|c|c|}
\hline Geometry: & $\begin{array}{l}\text { SOURCE: } \\
\text { Spherical }\end{array}$ & $\begin{array}{l}\text { SHIELDS: } \\
\text { Spherica? }\end{array}$ & $\begin{array}{l}\text { MODE } \\
\text { IGEOM } \\
\text { ICONC } \\
\text { SFACT } \\
\text { ISPEC }\end{array}$ & $\begin{array}{l}=1 \\
=3 \\
=0 \\
=1.000 E+00 \\
=3\end{array}$ \\
\hline \multicolumn{5}{|c|}{ Dimensions, cm: } \\
\hline $\begin{array}{l}x=4 . \\
y=0 .\end{array}$ & $\begin{array}{l}+01 \\
+00\end{array}$ & & $\begin{array}{l}\text { SLTH } \\
\text { VOLUME }\end{array}$ & $\begin{array}{l}=0.000 E+00 \\
=1.414 E+04\end{array}$ \\
\hline$D=x$ & $M(T N)=3 . C$ & $E+01$ & NSHLD & $=1$ \\
\hline
\end{tabular}

Shield Thickness, cm:

1.500E+01

Integration Specification:

Buildup Data:

NTHETA $=0$

NPSI $=1$

Taylor Coefficient

For Shield: 1 
WHC-SD-WV-CN-001, Rev. 0
Attachment C
Page 64 of 69

DELR $=0.000 E+00$

Effective At. No. 10.2

Group Average Bremsstr. Source Total Energy Flux Exposure Energy MeV photons/sec photons/sec MeV/cm2/s Roentgens $/ \mathrm{hr}$

\begin{tabular}{rrrrrr}
1 & $1.500 \mathrm{E}-02$ & $2.115 \mathrm{E}+07$ & $2.832 \mathrm{E}+07$ & $0.000 \mathrm{E}+00$ & $0.000 \mathrm{E}+00$ \\
2 & $2.500 \mathrm{E}-02$ & $1.188 \mathrm{E}+07$ & $1.208 \mathrm{E}+07$ & $0.000 \mathrm{E}+00$ & $0.000 \mathrm{E}+00$ \\
3 & $3.500 \mathrm{E}-02$ & $7.033 \mathrm{E}+06$ & $4.022 \mathrm{E}+07$ & $0.000 \mathrm{E}+00$ & $0.000 \mathrm{E}+00$ \\
4 & $4.500 \mathrm{E}-02$ & $4.760 \mathrm{E}+06$ & $6.183 \mathrm{E}+06$ & $0.000 \mathrm{E}+00$ & $0.000 \mathrm{E}+00$ \\
5 & $5.500 \mathrm{E}-02$ & $3.460 \mathrm{E}+06$ & $4.936 \mathrm{E}+06$ & $0.000 \mathrm{E}+00$ & $0.000 \mathrm{E}+00$ \\
6 & $6.500 \mathrm{E}-02$ & $2.925 \mathrm{E}+06$ & $2.938 \mathrm{E}+06$ & $0.000 \mathrm{E}+00$ & $0.000 \mathrm{E}+00$ \\
7 & $7.500 \mathrm{E}-02$ & $2.624 \mathrm{E}+06$ & $2.642 \mathrm{E}+06$ & $0.000 \mathrm{E}+00$ & $0.000 \mathrm{E}+00$ \\
8 & $8.500 \mathrm{E}-02$ & $2.345 \mathrm{E}+06$ & $2.609 \mathrm{E}+06$ & $0.000 \mathrm{E}+00$ & $0.000 \mathrm{E}+00$ \\
9 & $9.500 \mathrm{E}-02$ & $2.090 \mathrm{E}+06$ & $2.098 \mathrm{E}+06$ & $0.000 \mathrm{E}+00$ & $0.000 \mathrm{E}+00$ \\
10 & $1.500 \mathrm{E}-01$ & $9.403 \mathrm{E}+06$ & $1.146 \mathrm{E}+07$ & $1.793 \mathrm{E}+01$ & $3.098 \mathrm{E}-05$ \\
11 & $2.500 \mathrm{E}-01$ & $3.040 \mathrm{E}+06$ & $3.348 \mathrm{E}+06$ & $1.452 \mathrm{E}+01$ & $2.845 \mathrm{E}-05$ \\
12 & $3.500 \mathrm{E}-01$ & $1.465 \mathrm{E}+06$ & $1.475 \mathrm{E}+06$ & $1.076 \mathrm{E}+01$ & $2.216 \mathrm{E}-05$ \\
13 & $4.750 \mathrm{E}-01$ & $1.082 \mathrm{E}+06$ & $1.264 \mathrm{E}+06$ & $1.434 \mathrm{E}+01$ & $2.925 \mathrm{E}-05$ \\
14 & $6.500 \mathrm{E}-01$ & $6.306 \mathrm{E}+05$ & $4.121 \mathrm{E}+08$ & $6.417 \mathrm{E}+03$ & $1.335 \mathrm{E}-02$ \\
15 & $8.250 \mathrm{E}-01$ & $2.290 \mathrm{E}+05$ & $9.969 \mathrm{E}+05$ & $1.858 \mathrm{E}+01$ & $3.716 \mathrm{E}-05$ \\
16 & $1.000 \mathrm{E}+00$ & $1.513 \mathrm{E}+05$ & $1.430 \mathrm{E}+06$ & $3.323 \mathrm{E}+01$ & $6.413 \mathrm{E}-05$ \\
17 & $1.225 \mathrm{E}+00$ & $7.494 \mathrm{E}+04$ & $1.864 \mathrm{E}+06$ & $5.372 \mathrm{E}+01$ & $9.884 \mathrm{E}-05$ \\
18 & $1.475 \mathrm{E}+00$ & $2.378 \mathrm{E}+04$ & $1.587 \mathrm{E}+05$ & $5.552 \mathrm{E}+00$ & $9.772 \mathrm{E}-06$ \\
19 & $1.700 \mathrm{E}+00$ & $5.262 \mathrm{E}+03$ & $5.274 \mathrm{E}+03$ & $2.204 \mathrm{E}-01$ & $3.769 \mathrm{E}-07$ \\
20 & $1.900 \mathrm{E}+00$ & $1.067 \mathrm{E}+03$ & $2.687 \mathrm{E}+04$ & $1.267 \mathrm{E}+00$ & $2.104 \mathrm{E}-06$ \\
21 & $2.100 \mathrm{E}+00$ & $5.958 \mathrm{E}+01$ & $6.610 \mathrm{E}+01$ & $3.456 \mathrm{E}-03$ & $5.530 \mathrm{E}-09$ \\
22 & $2.300 \mathrm{E}+00$ & $6.533 \mathrm{E}-04$ & $6.533 \mathrm{E}-04$ & $3.775 \mathrm{E}-08$ & $5.813 \mathrm{E}-14$ \\
23 & $2.500 \mathrm{E}+00$ & $3.225 \mathrm{E}-04$ & $8.590 \mathrm{E}-04$ & $5.439 \mathrm{E}-08$ & $8.268 \mathrm{E}-14$ \\
24 & $2.700 \mathrm{E}+00$ & $1.409 \mathrm{E}-04$ & $2.271 \mathrm{E}+02$ & $1.560 \mathrm{E}-02$ & $2.309 \mathrm{E}-08$ \\
25 & $3.000 \mathrm{E}+00$ & $5.780 \mathrm{E}-05$ & $7.114 \mathrm{E}-03$ & $5.458 \mathrm{E}-07$ & $7.805 \mathrm{E}-13$ \\
26 & $3.600 \mathrm{E}+00$ & $0.000 \mathrm{E}+00$ & $0.000 \mathrm{E}+00$ & $0.000 \mathrm{E}+00$ & $0.000 \mathrm{E}+00$ \\
27 & $4.500 \mathrm{E}+00$ & $0.000 \mathrm{E}+00$ & $0.000 \mathrm{E}+00$ & $0.000 \mathrm{E}+00$ & $0.000 \mathrm{E}+00$ \\
30 & $5.500 \mathrm{E}+00$ & $0.000 \mathrm{E}+00$ & $0.000 \mathrm{E}+00$ & $0.000 \mathrm{E}+00$ & $0.000 \mathrm{E}+00$ \\
\hline $\mathrm{T}$ & $6.600 \mathrm{TAL}+00$ & $0.000 \mathrm{E}+00$ & $0.000 \mathrm{E}+00$ & $0.000 \mathrm{E}+00$ & $0.000 \mathrm{E}+00$ \\
& $8.600 \mathrm{E}+00$ & $0.000 \mathrm{E}+00$ & $0.000 \mathrm{E}+00$ & $0.000 \mathrm{E}+00$ & $0.000 \mathrm{E}+00$ \\
\hline $\mathrm{N}$ & & $7.438 \mathrm{E}+07$ & $5.362 \mathrm{E}+08$ & $6.587 \mathrm{E}+03$ & $1.367 \mathrm{E}-02$
\end{tabular}




\section{WHC-SD-WV-CN-001, Rev.0 Attachment $\mathrm{C}$ Page 65 of 69}

1 WV source for $1 \mathrm{~g}$ glass, $15.0 \mathrm{~cm}$ rad, dose $45 \mathrm{~cm}$ from cent. Brems. generation in GLASS

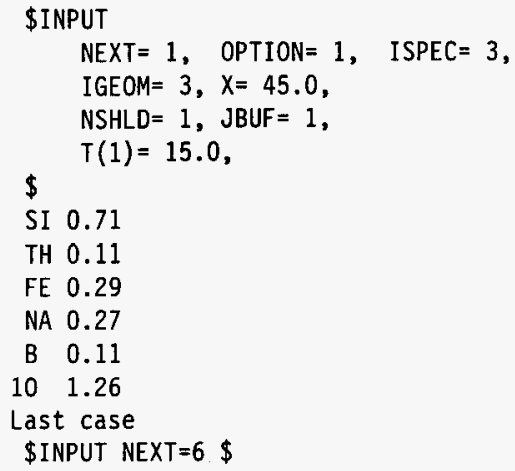


ISOSHLD 4.0

UNIX/IBM PC (September 1994)

Radiation Physics \& Shielding

Westinghouse Handford Company

Richland, WA 99352

Initializing photon 1ibrary: REAC*4 8/19/92 14:46:17

Initializing mass attenuation library: attnuate.lib

SHIELD COMPOSITION, $g / c c$

Shield 1 Shield 2 Shield 3 Shield 4 Shield 5

$\begin{array}{ll}\text { FE } & 3.450 \mathrm{E}-02 \\ \mathrm{~N} & 2.500 \mathrm{E}-02 \\ \mathrm{NA} & 6.040 \mathrm{E}-02 \\ \mathrm{TH} & 1.360 \mathrm{E}-02 \\ \mathrm{C} & 3.860 \mathrm{E}-02 \\ \mathrm{H} & 7.670 \mathrm{E}-02 \\ \mathrm{~B} & 3.680 \mathrm{E}-02 \\ \mathrm{P} & 4.700 \mathrm{E}-03 \\ \text { SI } & 8.510 \mathrm{E}-02 \\ 0 & 9.255 \mathrm{E}-01\end{array}$

>Retrieving data from ISODAT.QQQ

SRC FOR 1 cC of WV (glass) FEED

Feb 3,95 15:23:23 lass) FEED

Scale factor $=1.000 E+00$

Isotopic Source Term (unscaled)

$\begin{array}{llllll}\text { FE- 55 } & 1.200 E-07 & \text { CO- } 60 & 2.950 E-07 & \text { NI- 59 } & 8.920 E-08 \\ \text { SE- 79 } & 3.110 E-09 & \text { SR- 90 } & 4.890 E-03 & \text { Y }-90 & 4.890 E-03 \\ \text { ZR- } 93 & 2.290 E-07 & \text { NB- } 93^{*} & 1.710 E-07 & \text { TC- } 99 & 1.020 E-07\end{array}$




\section{WHC-SD-WV-CN-001, Rev.0 \\ Attachment $\mathrm{C}$ \\ Page 67 of 69}

$\begin{array}{llllll}\text { RU-106 } & 2.140 E-10 & \text { RH-106 } & 1.940 E-10 & \text { PD-107 } & 9.280 E-09 \\ \text { CD-113* } & 1.490 E-06 & \text { SN-121* } & 1.350 E-08 & \text { SN-126 } & 8.770 E-08 \\ \text { SB-125 } & 1.360 E-06 & \text { SB-126 } & 1.230 E-08 & \text { SB-126* } & 8.770 E-08 \\ \text { TE-125* } & 3.510 E-07 & \text { I }-129 & 1.520 E-10 & \text { CS-134 } & 5.890 E-07 \\ \text { CS-135 } & 1.380 E-07 & \text { CS-137 } & 5.380 E-03 & \text { BA-137* } & 5.090 E-03 \\ \text { CE-144 } & 2.620 E-12 & \text { PR-144 } & 2.630 E-12 & \text { PM-146 } & 4.280 E-09 \\ \text { PM-147 } & 1.510 E-05 & \text { SM-151 } & 6.780 E-05 & \text { EU-152 } & 2.260 E-07 \\ \text { EU-154 } & 4.960 E-05 & \text { EU-155 } & 8.680 E-06 & \text { TL-207 } & 7.920 E-09 \\ \text { TL-208 } & 2.520 E-09 & \text { PB-209 } & 1.810 E-10 & \text { PB-211 } & 7.950 E-09 \\ \text { PB-212 } & 7.020 E-09 & \text { BI-211 } & 7.950 E-09 & \text { BI-212 } & 7.020 E-09 \\ \text { BI-213 } & 1.810 E-10 & \text { P0-215 } & 7.950 E-09 & \text { PO-216 } & 7.020 E-09 \\ \text { RN-219 } & 7.950 E-09 & \text { RN-220 } & 7.020 E-09 & \text { FR-221 } & 1.810 E-10 \\ \text { FR-223 } & 1.100 E-10 & \text { RA-223 } & 7.950 E-09 & \text { RA-224 } & 7.020 E-09 \\ \text { RA-225 } & 1.810 E-10 & \text { RA-228 } & 1.330 E-09 & \text { AC-225 } & 1.810 E-10 \\ \text { AC-227 } & 7.950 E-09 & \text { AC-228 } & 1.330 E-09 & \text { TH-227 } & 7.830 E-09 \\ \text { TH-228 } & 7.020 E-09 & \text { TH-229 } & 1.810 E-10 & \text { TH-230 } & 4.940 E-11 \\ \text { TH-231 } & 7.970 E-11 & \text { TH-232 } & 1.380 E-09 & \text { TH-234 } & 6.720 E-10 \\ \text { PA-231 } & 1.280 E-08 & \text { PA-233 } & 1.980 E-08 & \text { PA-234* } & 6.720 E-10 \\ \text { U -232 } & 5.540 E-09 & \text { U -233 } & 7.610 E-09 & \text { U }-234 & 3.640 E-09 \\ \text { U -235 } & 7.970 E-11 & \text { U -236 } & 2.340 E-10 & \text { U -238 } & 6.720 E-10 \\ \text { NP-236 } & 7.980 E-09 & \text { NP-237 } & 1.980 E-08 & \text { NP-239 } & 2.920 E-07 \\ \text { PU-236 } & 7.100 E-10 & \text { PU-238 } & 6.670 E-06 & \text { PU-239 } & 1.370 E-06 \\ \text { PU-240 } & 1.010 E-06 & \text { PU-241 } & 5.090 E-05 & \text { PU-242 } & 1.370 E-09 \\ \text { AM-241 } & 4.510 E-05 & \text { AM-242 } & 2.420 E-07 & \text { AM-242* } & 2.430 E-07 \\ \text { AM-243 } & 2.920 E-07 & \text { CM-242 } & 2.010 E-07 & \text { CM-243 } & 9.760 E-08 \\ \text { CM-244 } & 5.120 E-06 & \text { CM-245 } & 7.430 E-40 & \text { CM-246 } & 8.500 E-11\end{array}$

Case Title: Brems. generation in FEED

\begin{tabular}{|c|c|c|c|c|}
\hline Geometry: & $\begin{array}{l}\text { SOURCE: } \\
\text { Spherical }\end{array}$ & $\begin{array}{l}\text { SHIELDS: } \\
\text { Spherical }\end{array}$ & $\begin{array}{l}\text { MODE } \\
\text { IGEOM } \\
\text { ICONC } \\
\text { SFACT } \\
\text { ISPEC }\end{array}$ & $\begin{array}{l}=1 \\
=3 \\
=0 \\
=1.000 E+00 \\
=3\end{array}$ \\
\hline \multicolumn{5}{|c|}{ Dimensions, cm: } \\
\hline $\begin{array}{l}X=4 . \\
Y=0 .\end{array}$ & $\begin{array}{l}+01 \\
+00\end{array}$ & & VOLUME & $\begin{array}{l}=0.000 \mathrm{E}+00 \\
=1.414 \mathrm{E}+04\end{array}$ \\
\hline
\end{tabular}

Shield Thickness, cm: 1.500E+01 
WHC-SD-WV-CN-001, Rev.0

Attachment $\mathrm{C}$

Page 68 of 69

Integration Specification:

NTHETA $=0$

NPSI $=1$

DELR $=0.000 E+00$
Buildup Data:

Taylor Coefficient

For Shield: 1

Effective At. No. 4.6
Group Average Bremsstr. Source Total Energy Flux Exposure Energy MeV photons $/ \mathrm{sec}$ photons $/ \mathrm{sec} \mathrm{MeV} / \mathrm{cm} 2 / \mathrm{s}$ Roentgens $/ \mathrm{hr}$

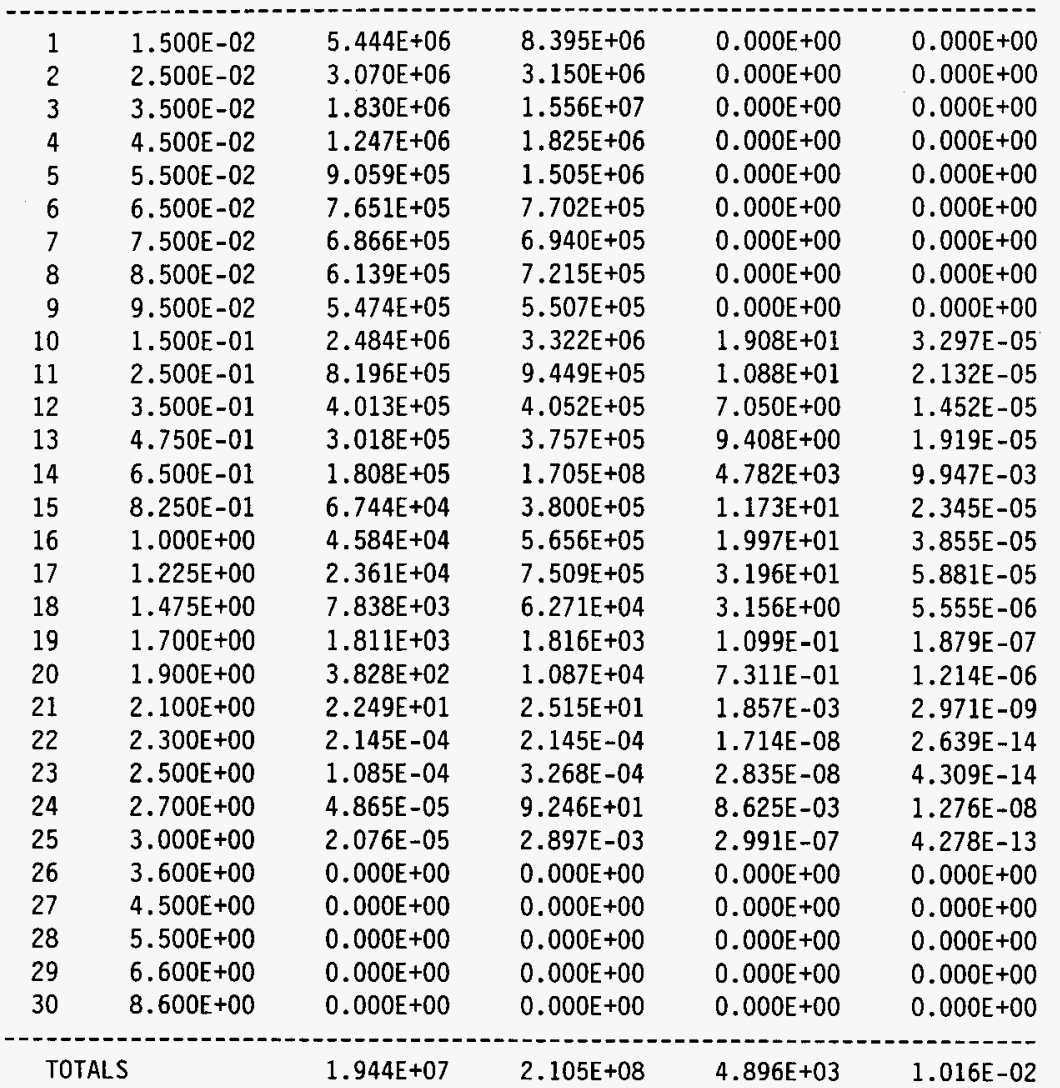


$\star * *$ End of Run $* * * *$

1 WV SRC for $1 \mathrm{cc}$ (glass) FEED, $15.0 \mathrm{~cm}$ rad, dose $45 \mathrm{~cm}$ from cent. Brems. generation in FEED

\$INPUT

NEXT $=1, \quad O P T I O N=1, \quad$ ISPEC $=3$,

IGEOM $=3, X=45.0$,

NSHLD $=1, J B U F=1$,

$\$$

$T(1)=15.0$,

FE $\quad 0.0345$

N 0.0250

NA 0.0604

TH 0.0136

C $\quad 0.0386$

$\begin{array}{ll}\text { H } & 0.0767\end{array}$

B $\quad 0.0368$

$\begin{array}{ll}P & 0.0047\end{array}$

SI 0.0851

$10 \quad 0.9255$

Last case

\$INPUT NEXT=6 \$ 


\begin{tabular}{|c|c|c|c|c|c|}
\hline \multirow{2}{*}{$\begin{array}{l}\text { To } \\
\text { Distribution }\end{array}$} & \multirow{2}{*}{\multicolumn{3}{|c|}{$\begin{array}{l}\text { From } \\
\text { Nuclear Physics and Shielding }\end{array}$}} & \multicolumn{2}{|l|}{ Page 1 of 1} \\
\hline & & & & \multicolumn{2}{|c|}{ Date $04 / 30 / 96$} \\
\hline \multicolumn{4}{|l|}{ Project Title/Work Order } & \multicolumn{2}{|c|}{ EDT No. 603240} \\
\hline \multicolumn{4}{|c|}{$\begin{array}{l}\text { MCNP Calculational Models of West Valley Vitrification Cell and } \\
\text { Point-Kernel Dose Contour Plots }\end{array}$} & \multicolumn{2}{|c|}{ ECN No. N/A } \\
\hline Name & $M S I N$ & $\begin{array}{c}\text { Text } \\
\text { With All } \\
\text { Attach. }\end{array}$ & Text Only & $\begin{array}{l}\text { Attach./ } \\
\text { Appendix } \\
\text { Only }\end{array}$ & $\begin{array}{l}\text { EDT/ECN } \\
\text { Oniy }\end{array}$ \\
\hline
\end{tabular}

J. Greenborg (2 copies)

HO-35 $X$

V. E. Roetman (10 copies)

HO-35 X

R. L. Simons

HO-35 X

Central files (Original +3 )

A3-88 $X$ 